\title{
Software Model-Checking as Cyclic-Proof Search
}

\author{
TAKESHI TSUKADA, Chiba University, Japan \\ HIROSHI UNNO, University of Tsukuba, Japan and RIKEN AIP, Japan
}

\begin{abstract}
This paper shows that a variety of software model-checking algorithms can be seen as proof-search strategies for a non-standard proof system, known as a cyclic proof system. Our use of the cyclic proof system as a logical foundation of software model checking enables us to compare different algorithms, to reconstruct well-known algorithms from a few simple principles, and to obtain soundness proofs of algorithms for free Among others, we show the significance of a heuristics based on a notion that we call maximal conservativity; this explains the cores of important algorithms such as property-directed reachability (PDR) and reveals a surprising connection to an efficient solver of games over infinite graphs that was not regarded as a kind of PDR.
\end{abstract}

CCS Concepts: - Theory of computation $\rightarrow$ Logic and verification; Program verification; Invariants.

Additional Key Words and Phrases: software model-checking, fixed-point logic, cyclic proof system, propertydirected reachability

ACM Reference Format:

Takeshi Tsukada and Hiroshi Unno. 2022. Software Model-Checking as Cyclic-Proof Search. Proc. ACM Program. Lang. 6, POPL, Article 63 (January 2022), 29 pages. https://doi.org/10.1145/3498725

\section{INTRODUCTION}

Software model-checkers [Beyer et al. 2018; Jhala and Majumdar 2009] are tools for verifying systems described by programs. They have been successfully applied to industrial software systems, in particular OS device drivers [Ball et al. 2004; Ball and Rajamani 2002; Khoroshilov et al. 2010]. To address the so-called "state explosion problem" in model checking real-world programs, the past decades have witnessed a significant development of state-space abstraction and refinement techniques including predicate abstraction [Ball et al. 2001; Graf and Saïdi 1997], CounterExampleGuided Abstraction Refinement (CEGAR) [Clarke et al. 2003], lazy abstraction [Beyer and Wendler 2012; Henzinger et al. 2004, 2002; McMillan 2006], and Property Directed Reachability (PDR) [Beyer and Dangl 2020; Birgmeier et al. 2014; Bradley 2011; Cimatti and Griggio 2012; Cimatti et al. 2014; Een et al. 2011; Hoder and Bjørner 2012; Komuravelli et al. 2014, 2013; Vizel and Gurfinkel 2014].

Software model-checkers can be classified by their target programming language and target properties. This paper mainly focuses on the safety verification problem for while languages.

The safety of a given program can be witnessed by an over-approximation of reachable states that does not intersect with the set of bad states. Such a desirable over-approximation can be characterized by the following three conditions: (1) it contains all initial states, (2) it is closed under the transition relation, and (3) it does not contain any bad state. Once a candidate of an approximation is given, it is relatively easy to check whether it satisfies the above conditions.

Authors' addresses: Takeshi Tsukada, Chiba University, Japan, tsukada@math.s.chiba-u.ac.jp; Hiroshi Unno, University of Tsukuba, Japan and RIKEN AIP, Japan, uhiro@cs.tsukuba.ac.jp.

This work is licensed under a Creative Commons Attribution 4.0 International License.

(c) 2022 Copyright held by the owner/author(s).

2475-1421/2022/1-ART63

https://doi.org/10.1145/3498725

Proc. ACM Program. Lang., Vol. 6, No. POPL, Article 63. Publication date: January 2022. 
Hence the most challenging part of the software model-checking is to find an appropriate overapproximation.

A modern software model checker guesses a candidate of over-approximation and iteratively refines it until a desirable one is found. Many papers were devoted to provide efficient procedures, including the above mentioned techniques for abstraction and refinement, to find an appropriate over-approximation, which vary in the structure of candidates and the candidate update method [Ball et al. 2001; Birgmeier et al. 2014; Cimatti and Griggio 2012; Cimatti et al. 2014; Henzinger et al. 2004, 2002; Hoder and Bjørner 2012; Komuravelli et al. 2014, 2013; McMillan 2006].

This paper aims to provide a unified account for a variety of approaches to software modelchecking in terms of logic, or more precisely, proof search. Since the notion of reachable states is an inductive notion, we need a proof system with (co-)induction.

A famous proof rules for reasoning about (co-)induction are based on pre- and/or post-fixed points (see, e.g., [Martin-Löf 1971]). However, they are not appropriate for the purpose of interpreting various abstraction and refinement techniques as proof-search strategies, because these proof rules are applicable only after appropriate pre- or post-fixed-points are found. Therefore the main process of software model-checking, trial-and-error search of appropriate over-approximation, has no logical interpretation.

We show that well-known procedures can naturally be seen as proof-search strategies of a nonstandard proof system, known as a cyclic proof system [Brotherston and Simpson 2011; Sprenger and Dam 2003]. Figure 1 illustrates a correspondence between McMillan's lazy abstraction with interpolants [McMillan 2006] and cyclic proof search. We do not explain the details here, which are the topic of Section 4, but we believe that the correspondence should be intuitively understandable.

Although the connection is somewhat expected, establishing a precise connection is not trivial. This is because the most natural logical expression of the software model-checking, based on the least-fixed-point characterization of reachable states, does not fit to practical algorithms (see Section 5). Our key observation to establish a tight connection between cyclic proof search and software model-checking is that we use the dual notion, safe states.

Our framework covers important procedures including variants of PDR [Cimatti and Griggio 2012; Hoder and Bjørner 2012], where we identify the significance of an underlying principle we call maximal conservativity of refinement. It turns out that the principle has also been adopted implicitly by highly efficient refinement algorithms, partially witnessing the usefulness of the principle in practice: one based on Craig interpolation [Craig 1957] for a variant of PDR [Vizel and Gurfinkel 2014] and one based on quantified satisfaction [Farzan and Kincaid 2016] for a solver of games over infinite graphs [Farzan and Kincaid 2017]. To the best of our knowledge, we are the first to apply the maximal conservativity, as one of the fundamental principles of software model checking, to formalize and compare variants of PDR and McMillan's lazy abstraction within a unified framework. This allowed us to construct a counterexample for the refutational completeness of existing PDR variants [Hoder and Bjørner 2012; Komuravelli et al. 2014, 2016] and to obtain the first PDR variant with refutational completeness.

The logical foundation of software model checking based on cyclic proof search has the following advantages.

- It allows us to compare different approaches to software model checking. Different approaches are different proof-search strategies for the same goal sequent in the same proof system. It is even possible to compare a software model-checking algorithm with algorithms for other problems. As an example, we show that PDR shares the same heuristic based on the principle of maximal conservativity as the solver for infinite games [Farzan and Kincaid 2017]. 


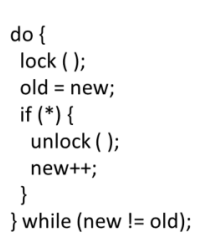

(a) program fragment

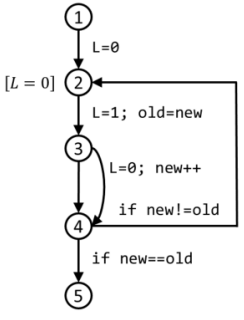

(b) control-flow graph

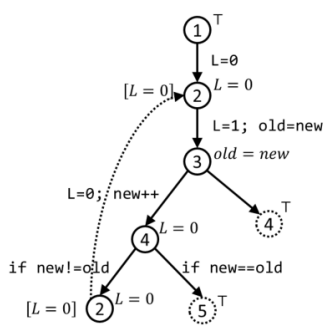

(c) intermediate state of IMPACT

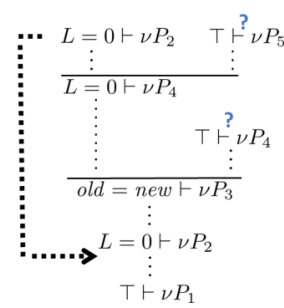

(d) a partial cyclic proof

Fig. 1. Correspondence between an intermediate state of McMillan's IMPACT and an intermediate state of cyclic-proof search. Figures (a-c) originate from [McMillan 2006]. (a) An example of a program. (b) The control flow graph of (a). The assertion is that $L=0$ at location 2. (c) An intermediate state of ImpACT. Nodes with the dotted line are needed to be expanded to confirm the safety. (d) An intermediate state of cyclic-proof search. The sequents with ? mark are open, which are not yet proved. For each location $i, v P_{i}$ is the formula corresponding to it; a sequent $\varphi \vdash v P_{i}$ corresponds to the node in (c) of location $i$ labeled with the formula $\varphi$.

- It allows us to reconstruct well-known algorithms from a few simple principles. This is because a proof system is demanding: use of a proof rule requires us to ensure side conditions. For example, an internal state of PDR is a sequence of formulas that satisfies certain conditions; these conditions indeed coincide with the side conditions of proof rules.

- We obtain soundness of model-checking algorithms for free. It is just a consequence of soundness of the proof system.

Note that the logical foundation goes beyond approaches [Bjørner et al. 2015; Flanagan 2004; Podelski and Rybalchenko 2007] based on constraint logic programming (CLP): they use CLP to capture mainly the process of modeling verification problems, while we use a cyclic-proof system to capture both processes of modeling and solving verification problems; we expect that the logical foundation based on cyclic proofs would also be convenient for developing new algorithms and heuristics. We shall briefly discuss some possible directions, though the detailed studies are left for future work. Furthermore, we expect that the logical foundation paves the way for transferring abstraction and refinement techniques studied in the software model checking community to cyclic proof-search, in particular, for finding necessary cut-formulas, which is considered essential for the success of proof-search because cyclic proof systems usually do not admit cut-elimination [Kimura et al. 2020; Masuoka and Tatsuta 2021].

Organization of this paper. The rest of the paper is organized as follows. Section 2 briefly reviews software model checking and two approaches: one is based on an over-approximation of reachable states and the other is based on a cyclic proof system. Section 3.1 discusses what is an appropriate goal sequent to establish the connection between cyclic proof search and software model-checking. Sections 3.2-6 exemplify the generality of the logical foundation based on cyclic proofs, by presenting proof-search strategies corresponding to well-known software model-checking techniques: symbolic execution, bounded model checking, predicate abstraction, lazy abstraction, and PDR. Section 7 shows a connection between an efficient procedure for game-solving and PDR. We discuss related work in Section 8 and conclude the paper with final remarks in Section 9. A longer version is available on the arXiv [Tsukada and Unno 2021]. 


\section{BACKGROUND}

\subsection{Definition of Software Model-Checking}

Recall that we have focused on verification of safety properties of while programs. A program induces a transition system with finite control states and infinite data, and we are interested in whether a bad state is reachable. For simplicity, we assume that the target program has exactly one control state. It is not difficult to apply the ideas of the paper to programs with multiple but finite control states, by introducing an auxiliary variable representing the current control state.

A transition system consists of a domain $\mathcal{D}$ of infinite data and a subset ${ }^{1} \iota \subseteq \mathcal{D}$ of initial states and a transition relation $\tau \subseteq \mathcal{D} \times \mathcal{D}$; an assertion is given as a subset $\alpha \subseteq \mathcal{D}$. A typical example of $\mathcal{D}$ is $\mathbb{Z}^{k}$ where $k$ is the number of variables in the program.

A state $x \in \mathcal{D}$ is reachable if there is a finite sequence $x_{0}, x_{1}, \ldots, x_{n}(n \geq 0)$ such that $\iota\left(x_{0}\right) \wedge$ $\bigwedge_{i=1}^{n} \tau\left(x_{i-1}, x_{i}\right) \wedge x_{n}=x$ holds. The safety verification problem asks whether, given a while program and an assertion, there is no reachable state that violates the assertion.

\subsection{Approach Based on Over-Approximation of Reachable States}

Unfortunately the set $R$ of reachable states is hard to compute, and modern model-checkers avoid the exact computation of $R$ by using an equational characterization of $R$. The set $R$ of reachable states satisfies the following condition:

$$
\iota(y) \vee(\exists x \cdot R(x) \wedge \tau(x, y)) \Leftrightarrow R(y) .
$$

Actually this condition characterizes the set of reachable states: it is the least solution of Equation 1. Defining a notion as the least solution of an equation in a certain class is called an inductive definition. So $R$ is an inductively defined predicate.

Thanks to the inductive definition of reachable states by Equation 1, it suffices to find any solution $R$ of Equation 1, which is not necessarily least, such that $R(x) \Rightarrow \alpha(x)$. If any solution $R$ of Equation 1 satisfies $R(x) \Rightarrow \alpha(x)$, then obviously so does the least solution of Equation 1 .

The condition can be further relaxed. It is well-known that the least solution of Equation 1 coincides with the least solution of

$$
\iota(y) \vee(\exists x \cdot R(x) \wedge \tau(x, y)) \Rightarrow R(y) .
$$

Theoretically the least solution can be computed by iteratively applying the predicate transformer $\mathcal{F}[\varphi](y):=\iota(y) \vee(\exists x . \varphi(x) \wedge \tau(x, y))$ to the empty set.

\subsection{Approach Based on a Proof System for Inductive Predicates}

The previous subsection reduces the safety verification problem to the entailment problem $R(x) \Rightarrow$ $\alpha(x)$, where $R$ is an inductively defined predicate. Hence a proof system for inductively defined predicates would serve as a basis for software model-checking.

Let us first fix notations. Assume an equation $P(x) \Leftrightarrow \delta[P](x)$, where $P$ occurs only positively in $\delta[P]$ (i.e. every occurrence of $P$ is under an even number of negation operators). Here $\delta$ is an unary predicate that defines $P$, which itself may depend on $P$; this dependency is written explicitly as $\delta[P]$. We write the least solution of this equation as $(\mu P)(x){ }^{2}$ where $\mu$ is the standard symbol for the least fixed-points.

This paper focuses on a cyclic proof system [Brotherston and Simpson 2011; Sprenger and Dam 2003]. It is an extension of the sequent calculus for the first-order classical logic, with two additional

\footnotetext{
${ }^{1}$ We shall identify a subset $A \subseteq X$ with a predicate over $X$ given by $A(x) \Leftrightarrow(x \in A)$.

${ }^{2}$ To simplify the notation, the defining equation is implicit. By explicitly writing the defining equation, $\mu P$ can be written as $\mu P . \lambda x . \delta[P](x)$.
} 


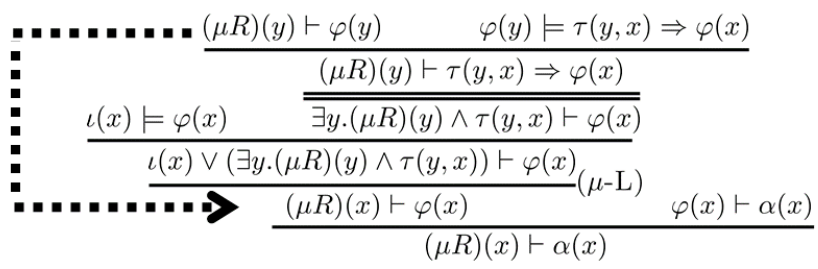

(a)

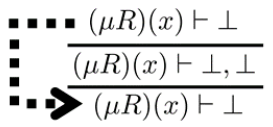

(b)

Fig. 2. Pre-proofs and the global trace condition. The preproof (a) satisfies the global trace condition, but the preproof (b) does not. So (a) is a cyclic proof but (b) is not. The inductive predicate $R$ is defined by Equation 1 .

mechanisms to deal with inductively defined predicates. The first one is the additional proof rules for inductively defined predicates:

$$
\frac{\delta[(\mu P)](x) \vdash \varphi(x)}{(\mu P)(x) \vdash \varphi(x)}(\mu-\mathrm{L}) \quad \frac{\varphi(x) \vdash \delta[(\mu P)](x)}{\varphi(x) \vdash(\mu P)(x)}(\mu-\mathrm{R})
$$

These rules just expand the definition of the predicate $\mu P$. For example, for the reachability predicate $\mu R$ defined by Equation 1, we have

$$
\frac{\iota(x) \vee(\exists y \cdot(\mu R)(y) \wedge \tau(y, x)) \vdash \varphi(x)}{(\mu R)(x) \vdash \varphi(x)}(\mu-\mathrm{L}) .
$$

The second mechanism is about the structure of proofs. In the cyclic proof system, a proof is not a tree but can have cycles; instead of proving the sequent of a leaf node, one can make a link to its ancestor with the same sequent.

A pre-proof is a proof-like tree whose leaves are either instances of the axiom rule or equipped with links to their ancestors. Figure 2 shows two examples of pre-proofs. Intuitively a link corresponds to a use of induction; for example, the proof of Figure 2(a) says that "we prove the sequent $(\mu R)(x) \vdash \varphi(x)$ at the second line from the bottom by induction on $x$ " and that "the leaf node $(\mu R)(y) \vdash \varphi(x)$ follows from the induction hypothesis."

Not all pre-proofs are valid; for example, the proof in Figure 2(b) has an invalid conclusion. Intuitively the invalidity is caused by a wrong use of the induction hypothesis. Recall that the induction hypothesis is applicable to only smaller elements, i.e. when proving $P(t)$, the induction hypothesis tells us $P(u)$ for $u<t$ but not for $u \geq t$. So if one applies the induction hypothesis to an element not smaller than $t$, it is a wrong proof. Figure 2(b) makes this kind of mistake.

A pre-proof is valid if it uses the "induction hypotheses" appropriately. The appropriateness in the context of cyclic proof systems is called the global trace condition. Because its definition is rather complicated (see [Brotherston and Simpson 2011]), we do not formally define the global trace condition. For pre-proofs appearing in this paper, the following condition is equivalent to the global trace condition:

If there is a link from a leaf $l$ to its ancestor $n,(\mu-\mathrm{L})$ rule is used in the path from $n$ to $l$.

Figure 2(a) satisfies the global trace condition in the above sense, but Figure 2(b) does not. A proof is a pre-proof that satisfies the global trace condition.

Example 2.1. Suppose that $\varphi$ is a solution of Equation 1 such that $\varphi(x) \Rightarrow \alpha(x)$. Then the cyclic proof of Figure 2(a) shows the sequent corresponding to the safety of the program. 


\subsection{Goal-Oriented Proof Search}

This paper studies a connection between software model-checking and the proof-search problem for a cyclic proof system, which asks to construct a proof of a given sequent. Proof search strategies of this paper are goal-oriented, i.e. a proof is constructed in a bottom-up manner. So an intermediate state is a proof-like structure in which some leaves are not yet proved. We call such an unproved leaf sequent an open sequent and a proof-like structure with open leaves a partial proof.

We fix a signature and a theory and assume that $\iota, \tau$ and $\alpha$ are formulas of the signature. We assume an external solver of the theory. We use $\models$ instead of $\vdash$ for the provability of a sequent handled by the external solver. For example, if a proof rule contains $\exists x . l(x) \wedge \tau(x, y) \vDash \alpha(y)$ as a premise, its validity should be checked by the external solver; our proof search strategies do not give any details of how to prove or disprove such sequents. We assume that a partial proof has no open sequent of the form $\varphi \vDash \psi$.

A partial proof is valid if all $=$-sequents in it are valid. We sometimes think of an invalid partial proof, which contains a false $\models$-sequent. A partial proof is either valid or invalid.

\section{BASIC NOTIONS OF SOFTWARE MODEL-CHECKING}

\subsection{The Goal Sequent}

As the first step to establish a connection between software model-checking and cyclic-proof search, this section describes the goal sequent corresponding to software model-checking. It might seem natural to use the sequent

$$
(\mu R)(y) \vdash \alpha(y) \quad \text { where } R(y) \Leftrightarrow(\iota(y) \vee(\exists x \cdot R(x) \wedge \tau(x, y))),
$$

which directly expresses that all reachable states satisfy the assertion $\alpha$. Unfortunately proof search for this sequent does not precisely correspond to existing procedures.

The key observation of this paper is that we should employ another logically-equivalent sequent, which uses the dual notion. We explain the dual notion from the view point of program verification, introducing the notion of safe states. We also briefly explain a proof system that handles coinductively defined predicates, the duals of inductively defined ones.

Safe states. From the view point of program verification, the dual formalization can be obtained by using the notion of safe states. A state is safe if the execution from the state will never reach a bad state. The safety verification problem can be alternatively formalized as the problem asking whether all initial states are safe.

Let us formally define safe states. A state $x$ is unsafe if there exists a sequence $x_{0}, \ldots, x_{n}(n \geq 0)$ such that $x=x_{0} \wedge \bigwedge_{i=1}^{n} \tau\left(x_{i-1}, x_{i}\right) \wedge \neg \alpha\left(x_{n}\right)$. A state is safe if it is not unsafe. The set $S$ of safe states has an equational characterization similar to the set of reachable states: it is the greatest solution of

$$
\alpha(x) \wedge(\forall y \cdot \tau(x, y) \Rightarrow S(y)) \Leftrightarrow S(x) .
$$

This kind of definition, specifying something as the greatest solution of an equation, is called coinductive definition, which is the dual of inductive definition. We write $v P$ for the greatest solution (provided that the equation for $P$ is understood). Following this notation, the set of safe states is $v S$.

Now the safety verification is reduced to the validity of the following sequent:

$$
\iota(x) \vdash(v S)(x) \quad \text { where } S(x) \Leftrightarrow(\alpha(x) \wedge(\forall y \cdot \tau(x, y) \Rightarrow S(y))) .
$$

This paper shows a tight connection between software model-checkers and cyclic-proof search for the above sequent. 
Cyclic proofs for coinductively defined predicates. The idea of cyclic proof systems is applicable to coinductively defined predicates. The proof system has the following rules:

$$
\frac{\delta[(v P)](x) \vdash \varphi(x)}{(v P)(x) \vdash \varphi(x)}(v-\mathrm{L}) \quad \frac{\varphi(x)+\delta[(v P)](x)}{\varphi(x) \vdash(v P)(x)}(v-\mathrm{R})
$$

where $P(x) \Leftrightarrow \delta[P](x)$ is the defining equation for $P$. A preproof is a proof if it satifies the following condition:

If there is a link from a leaf $l$ to its ancestor $n,(v-\mathrm{R})$ rule is used in the path from $n$ to $l$.

\subsection{Symbolic Execution}

The $(v-\mathrm{R})$ rule with some postprocessing corresponds to the symbolic execution of the one-step transition. This observation, which can be easily shown, is quite important because it is why software model-checking processes coincide with proof-search strategies of $\iota(x) \vdash(v P)(x)$. It also tells us why $(\mu P)(x) \vdash \alpha(x)$, which seems a more natural expression of the model-checking problem, does not suit for the purpose.

Definition 3.1 (Symbolic execution). Let $\varphi(x)$ be a formula denoting a set of states. Then the set of states after the transition $\tau$ from $\varphi$ can be represented by $\varphi^{\prime}(y):=\exists x . \varphi(x) \wedge \tau(x, y)$, and we write $\varphi \rightsquigarrow \varphi^{\prime}$. This transition on formulas is called symbolic execution.

Let us examine the connection between symbolic execution and $(v-\mathrm{R})$ rule. We discuss a proofsearch strategy for sequents of the form $\varphi(x) \vdash(v P)(x)$, which slightly generalize the goal sequent $\iota(x) \vdash(v P)(x)$.

Applying the $(v-\mathrm{R})$ rule to the sequent $\varphi(x) \vdash(v P)(x)$, we obtain

$$
\frac{\varphi(x) \vdash(\forall y \cdot \tau(x, y) \Rightarrow(v P)(y)) \wedge \alpha(x)}{\varphi(x) \vdash(v P)(x)}(v-\mathrm{R}) .
$$

Notice that the new open sequent, which is the premise of the above partial proof, has a shape much different from the goal sequent. For the purpose of proof search, it is convenient to restrict the shape of open sequents. We introduce the first proof-search principle of this paper.

Principle 1. Try to fit the shape of open sequents into the form $\varphi(x) \vdash(v P)(x)$.

We see that this principle leads to a proof strategy that corresponds to symbolic execution. Following Principle 1, we try to simplify the open sequent $\varphi(x) \vdash(\forall y . \tau(x, y) \Rightarrow(v P)(y)) \wedge \alpha(x)$ of the above partial proof. Fortunately its validity is equivalent to the validity of two simple sequents:

$$
\begin{aligned}
\varphi(x) & \vdash(\forall y . \tau(x, y) \Rightarrow(v P)(y)) \wedge \alpha(x) \\
& \Longleftrightarrow \quad \exists x . \varphi(x) \wedge \tau(x, y) \vdash(v P)(y) \quad \text { and } \quad \varphi(x) \vDash \alpha(x) .
\end{aligned}
$$

The right-to-left direction can be shown by the following partial proof:

$$
\begin{aligned}
& \frac{\frac{\vdots}{\varphi(x), \tau(x, y) \vdash \exists x . \varphi(x) \wedge \tau(x, y)} \quad \exists x . \varphi(x) \wedge \tau(x, y) \vdash(v P)(y)}{\frac{\varphi(x), \tau(x, y) \vdash(v P)(y)}{\varphi(x) \vdash \tau(x, y)} \text { (CUT) }} \\
& \frac{\frac{\overline{\varphi(x) \vdash \tau(x, y) \Rightarrow(v P)(y)}}{\varphi(x) \vdash \forall y \cdot \tau(x, y) \Rightarrow(v P)(y)} \quad \varphi(x) \vDash \alpha(x)}{\frac{\varphi(x) \vdash(\forall y \cdot \tau(x, y) \Rightarrow(v P)(y)) \wedge \alpha(x)}{\varphi(x) \vdash(v P)(x)}(v-\mathrm{R})}
\end{aligned}
$$




$$
\begin{aligned}
\frac{\varphi_{k+1}(x) \vdash(v P)(x) \quad \varphi_{k}(x) \vDash \alpha(x)}{\varphi_{k}(x) \vdash(v P)(x)} \text { (SE) } & \vdots \\
\vdots & \frac{\varphi_{2}(x) \vdash(v P)(x) \quad \varphi_{1}(x) \vDash \alpha(x)}{\frac{\varphi_{1}(x) \vdash(v P)(x)}{(\mathrm{SE})} \quad \varphi_{0}(x) \vDash \alpha(x)}
\end{aligned}
$$

Fig. 3. A partial proof corresponding to bounded model-checking. Here $\varphi_{0} \rightsquigarrow \varphi_{1} \rightsquigarrow \cdots \rightsquigarrow \varphi_{k} \rightsquigarrow \varphi_{k+1}$. The partial proof is valid if $\varphi_{i}(x) \vDash \alpha(x)$ for every $i=0, \ldots, k$.

(where the proof of $\varphi(x), \tau(x, y) \vdash \exists x . \varphi(x) \wedge \tau(x, y)$ is omitted). One of the leaves is an open sequent of the required form, and the other is fixed-point free and passed to an external solver. So this partial proof meets the criterion of Principle 1.

The above argument shows that the rule

$$
\frac{\exists x . \varphi(x) \wedge \tau(x, y) \vdash(v P)(y) \quad \varphi(x) \vDash \alpha(x)}{\varphi(x) \vdash(v P)(x)}
$$

is a derived rule. We call it the (precise) symbolic execution rule (SE). Since we are interested in proof-search strategies, this rule should be read bottom-up: In order to prove the satefy of $\varphi$, it suffices to (1) check if $\varphi$ does not violate the assertion, (2) do the symbolic execution $\varphi \rightsquigarrow \varphi^{\prime}$, and (3) show the safety of $\varphi^{\prime}$ by proving the sequent $\varphi^{\prime}(x) \vdash(\nu P)(x)$.

\subsection{Bounded Model-Checking}

The bounded model-checking problem [Biere et al. 1999] asks, given a bound $k$ and an initial state $\varphi_{0}$, whether a bad state is reachable from a state satisfying $\varphi_{0}$ by transitions of steps $\leq k$. More formally, it checks if $\varphi_{0} \rightsquigarrow \varphi_{1} \rightsquigarrow \cdots \rightsquigarrow \varphi_{k}$ implies $\varphi_{i}(x) \vDash \alpha(x)$ for every $i=0, \ldots, k$. If it is the case, we say $\varphi_{0}$ is safe within $k$ steps. The bounded model-checking problem is important because it is decidable in certain settings, and software model-checkers often have subprocedures corresponding to bounded model-checking.

In terms of cyclic-proof search, bounded model-checking is to construct a valid partial proof consisting only of (SE). By iteratively applying (SE), we obtain a (valid or invalid) partial proof in Figure 3. Here $\varphi_{0} \rightsquigarrow \varphi_{1} \rightsquigarrow \cdots \rightsquigarrow \varphi_{k} \rightsquigarrow \varphi_{k+1}$. This is a valid partial proof if $\varphi_{i}(x) \vDash \alpha(x)$ for every $i=0, \ldots, k$. Therefore bounded model-checking with bound $k$ is equivalent to the problem asking if the $k$ consecutive applications of (SE) to the corresponding sequent is a valid partial proof.

Proposition 3.2. The $k$ consecutive applications of $(S E)$ to $\varphi(x) \vdash(v X)(x)$ is a valid partial proof if and only if $\varphi$ is safe within $k$ steps.

Forward Criterion and States of Distance $k$. Sometimes postprocessing the result of bounded model-checking gives a proof of unbounded safety. We call the criterion in [Sheeran et al. 2000] based on a notion of distance (called forward diameter in [Sheeran et al. 2000]) forward criterion.

Let us fix the set $\iota$ of initial states. A reachable state $x$ is of distance $k$ if there exists a sequence $x_{0}, \ldots, x_{k}$ witnessing the reachability of $x$ from $\iota$ (i.e. $\left.\iota\left(x_{0}\right) \wedge \bigwedge_{i=1}^{k} \tau\left(x_{i-1}, x_{i}\right) \wedge\left(x_{k}=x\right)\right)$ and there is no shorter witness. If $\iota=\varphi_{0} \rightsquigarrow \varphi_{1} \rightsquigarrow \cdots \rightsquigarrow \varphi_{k}$, then the set of states of distance $k$ is represented by $\varphi_{k}(x) \wedge \bigwedge_{i=0}^{k-1} \neg \varphi_{i}(x)$. The forward criterion says that a program is safe if the program is safe with bound $k$ and the set of states of distance $k+1$ is empty.

The set of distance- $k$ states naturally appears in cyclic proof search. Recall that, in a cyclic proof system, a leaf sequent can be proved by putting a link to its ancestor labeled by the same 
judgement. For example, a proof of the open sequent $\varphi_{k+1}(x) \vdash(\nu P)(x)$ in Figure 3 is able to use the assumptions $\varphi_{i}(x) \vdash(v P)(x)(0 \leq i<k+1)$. The set of distance- $k$ states naturally arises when we try to exploit the assumptions to the full.

The idea is to split $\varphi_{k+1}(x)$ into $\varphi_{i}(x)(0 \leq i<k+1)$ and the remainder, say $\psi(x)$. Assume a formula $\psi$ such that $\varphi_{k+1}(x) \vDash \psi(x) \vee \bigvee_{0 \leq i<k+1} \varphi_{i}(x)$. Let us write $\Psi(x)$ for $\psi(x) \vee \bigvee_{0 \leq i<k+1} \varphi_{i}(x)$. The open sequent in the partial proof in Figure 3 can be expanded as

$$
\frac{\varphi_{k+1}(x) \vDash \Psi(x) \frac{\psi(x) \vdash(v P)(x) \quad \varphi_{0}(x) \vdash(v P)(x)}{\Psi(x) \vdash(v P)(x)}(\text { CUT }),}{\varphi_{k+1}(x) \vdash(v P)(x)}(\vee-\mathrm{L}) \times(k+1)
$$

which has $k+1$ open sequents. All but one open sequent can be immediately proved by links to ancestors; the only remaining open sequent is $\psi(x) \vdash(v P)(x)$. Since $\psi$ appears in a contra-variant position, a stronger $\psi$ is more desirable. The strongest $\psi$ that satisfies the requirement is given by $\psi(x) \Leftrightarrow\left(\varphi_{k+1}(x) \wedge \bigwedge_{0 \leq i<k+1} \neg \varphi_{i}(x)\right)$. This is exactly the set of distance- $(k+1)$ states. In particular, if the forward criterion holds, one can choose $\psi(x)$ as $\perp$. In this case, the remaining open sequent is $\perp \vdash(\nu P)(x)$, which is trivially provable independent of $P$; hence we complete the proof of $\varphi_{0}(x) \vdash(v P)(x) .^{3}$

\subsection{Predicate Abstraction}

As shown in the previous section, (CUT) plays an important role in the construction of a cyclic proof. Let us introduce the derived rule

$$
\frac{\psi(x) \vdash(v P)(x) \quad \exists y \cdot \varphi(y) \wedge \tau(y, x) \vDash \psi(x) \quad \varphi(x) \vDash \alpha(x)}{\varphi(x) \vdash(v P)(x)} \text { (SE+Cut), }
$$

which can be proved by

$$
\frac{\exists x . \varphi(x) \wedge \tau(x, y) \vDash \psi(y) \quad \psi(x) \vdash(v P)(x)}{\frac{\exists x . \varphi(x) \wedge \tau(x, y) \vdash(v P)(y)}{\varphi(x) \vdash(v P)(x)}(\mathrm{CUT}) \quad \varphi(x) \vDash \alpha(x)} \text { (SE). }
$$

We call $\psi$ in (SE+CUT) the cut formula.

The choice of the cut formula is quite important. If it is too strong, it is not an invariant; if it is too weak, the sequent $\psi(x) \vdash(v P)(x)$ becomes invalid.

Predicate abstraction [Ball et al. 2001; Graf and Saïdi 1997] is a heuristic for the choice of the cut formula. The idea is to further restrict the shape of open sequents: an open sequent must be of the form $\vartheta(x) \vdash(v P)(x)$ where $\vartheta$ comes from a finite set of predicates, say $\Xi$. Suppose that $\Xi$ is closed under conjunction. In order to avoid making the open sequent invalid, the best choice of the cut formula is the strongest formula in $\Xi$.

This idea leads to the following principle.

Principle 2. Use (SE+CUT). Choose the strongest formula $\psi \in \Xi$ satisfying $\exists x . \varphi(x) \wedge \tau(x, y) \vDash$ $\psi(y)$ as the cut formula.

The proof-search procedure following Principles 1 and 2 terminates. Since $\Xi$ is finite, the length of the path in a partial proof is bounded by the number of candidate formulas; if it exceeds, then a sequent appears twice in the path and thus putting a link completes the proof.

\footnotetext{
${ }^{3}$ Actually what we obtained is a pre-proof, i.e. a proof-like structure with cycles, and we should check the global trace condition to conclude that it is a proof. To see that the pre-proof satisfies the global trace condition, recall that (SE) is a derived rule using $(v-\mathrm{R})$ rule.
} 


\begin{tabular}{l|l} 
IMPACT & Cyclic proof \\
\hline program unwinding & partial proof \\
$\quad$ safe, well-labeled - & valid - \\
$\quad$ safe, well-labeled, complete - & cyclic proof \\
covering relation & collection of links \\
EXPAND procedure & application of (SE+CUT)
\end{tabular}

Table 1. An Iмpact-Cyclic-proof dictionary.

$$
\begin{aligned}
& \begin{array}{r}
\varphi_{n+1}(x) \vdash(v P)(x) \quad \exists x . \varphi_{n}(x) \wedge \tau(x, y) \vDash \varphi_{n+1}(y) \\
\hline \varphi_{n}(x) \vdash(v P)(x)
\end{array}
\end{aligned}
$$

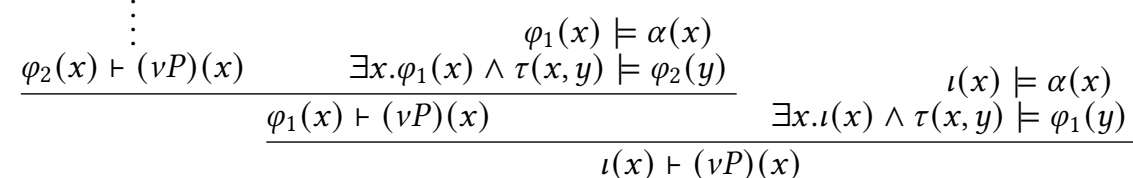

$$
\begin{aligned}
& \mathrm{O}_{\iota} \mathrm{O}_{\varphi_{1}} \mathrm{O}_{\varphi_{2}} \cdots \cdots \mathrm{O}_{\varphi_{k+1}}
\end{aligned}
$$

Fig. 4. A partial proof (above) and the corresponding internal state of ІмРАст (below). All rules used in the partial proof are (SE+CUT).

\section{LAZY ABSTRACTION WITH INTERPOLANTS: IMPACT}

This section explains another proof-search strategy that corresponds to the software model-checker IMPACT developed by McMillan [McMillan 2006]. For readers familiar with IMPACT, Table 1 gives a dictionary of terminologies of IMPACт [McMillan 2006] and cyclic-proof search and Figure 4 illustrates the correspondence between internal states of IмРАCт (called program unwinding) and partial proofs.

A significant difference from the previously discussed proof-search strategies is that IMPACT does not hesitate to generate an invalid sequent. The difference allows us to simplify the strategy of the cut-formula choice. Recall that the only requirement for the cut formula $\psi$ in (SE+CUT) rule is $\exists x . \varphi(x) \wedge \tau(x, y) \vdash \psi(y)$, and no upper bound is given. IMPACT tentatively chooses $\mathrm{T}$ as the cut formula.

Principle 3. Use (SE+CUt). Tentatively choose $T$ as the cut formula.

Of course, $\mathrm{T}$ is usually too weak and the open sequent $\mathrm{T} \vdash(v P)(x)$ is often invalid. The invalidity of the sequent is revealed by applying (SE+CUT) again:

$$
\frac{\mathrm{T \vdash}(v P)(x) \quad \exists x . \top \wedge \tau(x, y) \vDash \top \quad \mathrm{T} \vDash \alpha(x)}{\frac{\mathrm{T} \vdash(v P)(x)}{} \exists x . l(x) \wedge \tau(x, y) \vDash \top \quad \iota(x) \vDash \alpha(x)}
$$

This partial proof is invalid as $T \models \alpha(x)$ does not hold (otherwise the verification problem is trivial). IMPACT tries to fix an invalid partial proof by strengthening the cut formulas, following the next principle. ${ }^{4}$

Principle 4. When the partial proof is found to be invalid, replace each cut formula $\varphi_{i}(x)$ with $\varphi_{i}(x) \wedge Q_{i}(x)$, where $Q_{i}$ is a fresh predicate variable, and solve the constraints on $Q_{i}$.

\footnotetext{
${ }^{4}$ See Remark 1 for differences between IMPACт and the proof-search strategy following Principle 4.
} 
If the current partial proof is that in Figure 4, cut-formulas $\varphi_{1}, \ldots, \varphi_{n}$ must be strengthened if $\varphi_{n}(x) \not k \alpha(x)$. For simplicity, we assume $\varphi_{i}(x) \vDash \alpha(x)$ for $i<n$. We replace $\varphi_{i}(x)$ in the partial proof with $\varphi_{i}(x) \wedge Q_{i}(x)(1 \leq i \leq n)$, where $Q_{1}, \ldots, Q_{n}$ are distinct predicate variables. The side conditions $\varphi_{i}(x) \vDash \alpha(x)$ and $\exists x . \varphi_{i}(x) \wedge \tau(x, y) \vDash \varphi_{i+1}(x)$ are transformed into $\varphi_{i}(x) \wedge Q_{i}(x) \vDash \alpha(x)$ and $\exists x . \varphi_{i}(x) \wedge Q_{i}(x) \wedge \tau(x, y) \vDash \varphi_{i+1}(y) \wedge Q_{i+1}(y)$. Slightly simplifying the constraints by using $\exists x . \varphi_{i}(x) \wedge \tau(x, y) \vDash \varphi_{i+1}(y)(i=0, \ldots, n)$ and $\varphi_{i}(x) \vDash \alpha(x)(i=0, \ldots, n-1)$, we obtain the following constraint set:

$$
\begin{aligned}
\iota\left(x_{0}\right) \wedge \tau\left(x_{0}, x_{1}\right) & \Rightarrow Q_{1}\left(x_{1}\right), \\
Q_{i}\left(x_{i}\right) \wedge\left(\varphi_{i}\left(x_{i}\right) \wedge \tau\left(x_{i}, x_{i+1}\right)\right) & \Rightarrow Q_{i+1}\left(x_{i+1}\right), \\
Q_{n}\left(x_{n}\right) \wedge \varphi_{n}\left(x_{n}\right) & \Rightarrow \alpha\left(x_{n}\right)
\end{aligned}
$$

If a solution $\left(\psi_{i}\right)_{i=1, \ldots, n}$ for $\left(Q_{i}\right)_{i}$ is given, we can fix the partial proof by replacing $\varphi_{i}$ with $\varphi_{i} \wedge \psi_{i}$. A solution of the constraints is known as an interpolant.

Definition 4.1. Given a sequence $\vec{\vartheta}=\vartheta_{1} \vartheta_{2} \ldots \vartheta_{n+1}$ of formulas, its interpolant is a sequence $\vec{\psi}=\psi_{0} \psi_{1} \ldots \psi_{n+1}$ of formulas that satisfies the following conditions: (1) $\psi_{0}=\mathrm{T}$ and $\psi_{n+1}=\perp$, (2) $\vDash \psi_{i-1} \wedge \vartheta_{i} \Rightarrow \psi_{i}$ for every $1 \leq i \leq n$, and $(3) \mathrm{fv}\left(\psi_{i}\right) \subseteq \mathrm{fv}\left(\vartheta_{1}, \ldots, \vartheta_{i}\right) \cap \mathrm{fv}\left(\vartheta_{i+1}, \ldots, \vartheta_{n+1}\right)$ for every $1 \leq i \leq n$.

A solution of the above constraint is an interpolant of $\vec{\vartheta}$ where $\vartheta_{1}:=\iota\left(x_{0}\right) \wedge \tau\left(x_{0}, x_{1}\right), \vartheta_{i+1}:=$ $\varphi_{i}\left(x_{i}\right) \wedge \tau\left(x_{i}, x_{i+1}\right)$ for $i=1, \ldots, n-1$, and $\vartheta_{n+1}=\varphi_{n}\left(x_{n}\right) \wedge \neg \alpha\left(x_{n}\right)$.

The termination condition of IMPACT is also closely related to cyclic proofs. IMPACT terminates if $\varphi_{i}(x) \vDash \varphi_{j}(x)$ for some $i>j$. Then replacing the partial proof above $\varphi_{i}(x) \vdash(v P)(x)$ with

$$
\frac{\varphi_{i}(x) \vDash \varphi_{j}(x) \quad \varphi_{j}(x) \vdash(v P)(x)}{\varphi_{i}(x) \vdash(v P)(x)}(\mathrm{CUT})
$$

and making a link from $\varphi_{j}(x) \vdash(v P)(x)$ to the ancestor yields a cyclic proof.

In summary, IмРАст can be seen as a cyclic-proof search strategy following Principles 1, 3 and 4.

Remark 1. We discuss differences between IмPACT and the proof-search strategy following Principles 1, 3 and 4. First the proof-search strategy does not cover an important heuristic, known as forced covering, which tries to refine predicates so that one can make a cycle from a certain node to another; this feature can be understood as an additional proof-search rule. Second IMPACT computes an interpolant of a sequence slightly different from $\vec{\vartheta}$ : the sequence $\vec{\vartheta}$ contains the information of the current cut-formulas $\varphi_{i}$. Theoretically, the difference does not affect the refinement process (modulo logical equivalence), since the interpolants are conjoined with the current cut-formulas. In practice, however, [Vizel and Gurfinkel 2014] claims that the use of the current cut-formulas is one of the keys to achieve their incremental interpolation-based model checking, which indeed shows an improvement over previous methods based on interpolation a la ІмРАCт. Third the actual implementation of IмPAст uses a special kind of interpolant, which follows Principle 5 below as well. ${ }^{5}$

Remark 2. One may notice that the combination of Principles 1, 2 and 4 (instead of 1, 3 and 4) would also be possible. The resulting cyclic-proof search strategy corresponds to the lazy abstraction in the style of BLAST [Henzinger et al. 2004, 2002].

\footnotetext{
${ }^{5}$ We are grateful to an annonimous reviewer who pointed out this point.
} 


\section{DUAL SEQUENT AND DUAL IMPACT}

So far, we have discussed a relationship between cyclic-proof search for $\iota(x) \vdash(\nu P)(x)$ and software model-checking algorithms. As we emphasized in Section 3.1, the identification of the goal sequent $\iota(x) \vdash(v P)(x)$ corresponding to software model-checking is an important contribution of this paper, and once the goal sequent is appropriately set, the contents of Sections 3 and 4 are natural and expected, which one might find relatively straightforward.

This section briefly discusses proof-search for $(\mu R)(x) \vdash \alpha(x)$ (where $R$ is defined by Equation 1 ) from the view point of software model-checking, and shows how the choice of the goal sequent affects the whole picture.

We derive the counterparts of (SE) and (SE+CUT), called (BACKSE) and (BACKSE+CUT). We have

$$
\frac{\iota(x) \vee(\exists y \cdot(\mu R)(y) \wedge \tau(y, x)) \vdash \varphi(x)}{(\mu R)(x) \vdash \varphi(x)}(\mu-\mathrm{L})
$$

of which left-hand-side of $\vdash$ in the premise is a complicated formula containing a least fixed-point. Following the dual of Principle 1, moving logical connectives to the right-hand-side, we obtain the following derived rule:

$$
\frac{(\mu R)(x) \vdash \forall y \cdot \tau(x, y) \Rightarrow \psi(y) \quad \iota(x) \vDash \psi(x)}{(\mu R)(x) \vdash \psi(x)} \text { (BACKSE). }
$$

The formula $\forall y . \tau(x, y) \Rightarrow \psi(y)$ has a clear computational interpretation: it is the weakest precondition of $\psi$ with respect to $\tau$. This is the dual of the strongest postcondition; which coincides with the symbolic execution.

The duality becomes clearer in (BACKSE+CUT)

$$
\frac{(\mu R)(x) \vdash \varphi(x) \quad \varphi(x) \vDash \forall y \cdot \tau(x, y) \Rightarrow \psi(y) \quad \iota(x) \vDash \psi(x)}{(\mu R)(x) \vdash \psi(x)} \text { (BAскSE+Cut) }
$$

which can be proved by

$$
\frac{(\mu R)(x) \vdash \varphi(x) \quad \varphi(x) \vDash \forall y \cdot \tau(x, y) \Rightarrow \psi(y)}{\frac{(\mu R)(x) \vdash \forall y \cdot \tau(x, y) \Rightarrow \psi(y)}{(\mathrm{CUT})} \quad l(x) \vDash \psi(x)} \text { (BACKSE). }
$$

Since $\varphi(x) \vDash \forall y \cdot \tau(x, y) \Rightarrow \psi(y)$ is equivalent to $\exists x . \varphi(x) \wedge \tau(x, y) \vDash \psi(y)$, (BACKSE+CuT) computes an over-approximation of the symbolic execution as in (SE+CUT). The only difference is the direction; in a partial proof for $(\mu R)(x) \vdash \psi(x)$, an execution starts from a leaf and ends at the root, which should be understood as an assertion.

So model-checking algorithms corresponding to proof-search for $(\mu R)(x) \vdash \psi(x)$ can be understood as procedures checking the backward (un)reachability. Here is a twist: the natural logical expression $(\mu R)(x) \vdash \psi(x)$ of the model-checking problem corresponds to the execution of programs in the unnatural direction. We think that this twist might be a reason why the connection between cyclic proofs and software model-checking hashad not yet been formally revealed despite that it is natural and expected.

\section{MAXIMAL CONSERVATIVITY AND PROPERTY-DIRECTED REACHABILITY}

Sections 4 reviewed McMillan's approach based on interpolants [McMillan 2006], as well as its variants, from the view point of cyclic proof search. His algorithm tentatively uses $T$ as the cutformula at the beginning and improves it using interpolants. The efficiency of the algorithm heavily relies on the quality of interpolants. 
This section introduces a heuristic, or a criterion, that determines what is a "better" interpolation. The criterion is conservativity: it prefers an interpolation that introduces smaller changes to the current partial proof. We study a variant of McMillan's algorithm that uses a maximally conservative interpolation instead of arbitrary ones and discuss its connection to the approach to software modelchecking called property directed reachability (or PDR) [Cimatti and Griggio 2012; Hoder and Bjørner 2012].

\subsection{Maximal Conservativity}

We introduce the key notion, maximal conservativity, to the heuristic studied in this section.

Let us first recall McMillan's algorithm [McMillan 2006], regarded as a goal-oriented proof search. The refinement process takes an invalid partial proof

$$
\begin{aligned}
& \begin{aligned}
\varphi_{n}(x) & \models \alpha(x) \\
\top \vdash(v P)(x) \quad \exists x \cdot \varphi_{n}(x) \wedge \tau(x, y) & \vDash \mathrm{T}
\end{aligned} \\
& \begin{aligned}
\varphi_{1}(x) & \models \alpha(x) \\
\varphi_{2}(x) \vdash(v P)(x) \quad \exists x . \varphi_{1}(x) \wedge \tau(x, y) & \models \varphi_{2}(y)
\end{aligned} \quad \iota(x) \vDash \alpha(x) \\
& \frac{\varphi_{1}(x) \vdash(v P)(x) \quad \exists x . l(x) \wedge \tau(x, y) \vDash \varphi_{1}(y)}{\iota(x) \vdash(v P)(x)}
\end{aligned}
$$

in which all judgements except for $\varphi_{n}(x) \vDash \alpha(x)$ are valid. It returns a valid proof of $\iota(x) \vdash(v P)(x)$ obtained by replacing each $\varphi_{i}$ with a stronger predicate $\varphi_{i}^{\prime}$.

We represent the above partial proof as a sequence $\varphi_{0}, \varphi_{1}, \ldots, \varphi_{n}$ of formulas (where $\varphi_{0}=\imath$ ). Then an input to the refinement process can be represented as a sequence $\varphi_{0}, \ldots, \varphi_{n}$ of formulas that satisfies

$$
\varphi_{0}=\iota \quad \text { and } \quad \varphi_{i}(x) \vDash \alpha(x) \text { and } \varphi_{i}(x) \wedge \tau(x, y) \vDash \varphi_{i+1}(y) \text { for } i=0, \ldots, n-1
$$

(recall that all judgements in the input partial proof are valid except for $\varphi_{n}(x) \vDash \alpha(x)$ ). The refinement process tries to construct a sequence $\varphi_{0}^{\prime}, \ldots, \varphi_{n}^{\prime}$ such that

$$
\begin{cases}\varphi_{0}^{\prime}=\iota & \\ \varphi_{i}^{\prime}(x) \vDash \varphi_{i}(x) & \text { for every } i=0, \ldots, n \\ \varphi_{i}^{\prime}(x) \wedge \tau(x, y) \vDash \varphi_{i+1}^{\prime}(y) & \text { for every } i=0, \ldots, n-1 \\ \varphi_{n}^{\prime}(x) \vDash \alpha(x) . & \end{cases}
$$

We call a sequence that satisfies Equation 5 a refinement of $\varphi_{0}, \ldots, \varphi_{n}$.

In McMillan's approach [McMillan 2006], we let $\varphi_{i}^{\prime}(x) \equiv \varphi_{i}(x) \wedge Q_{i}(x)(i=0, \ldots, n)$, where $Q_{i}$ is a fresh predicate variable, and solve the constraints

$$
\left\{\left(\varphi_{i}(x) \wedge Q_{i}(x)\right) \wedge \tau(x, y) \Rightarrow Q_{i+1}(y) \mid i=0, \ldots, n-1\right\} \cup\left\{Q_{0}=\mathrm{\top}, \varphi_{n}(x) \wedge Q_{n}(x) \Rightarrow \alpha(x)\right\}
$$

using an interpolating theorem prover, finding a suitable assignment for $\left(Q_{i}\right)_{0 \leq i \leq n}$. The constraint solving of (6) is a kind of interpolation problem, known as sequential interpolation [Henzinger et al. 2004; McMillan 2006], we call a solution $\left(Q_{i}\right)_{0 \leq i \leq n}$ an interpolant. Observe that this refinement process may change any formula in the current partial proof.

The heuristic studied in this section tries to minimize the changes. That means, it prefers an interpolant $\left(Q_{i}\right)_{0 \leq i \leq n}$ of (6) such that $Q_{i}=\top$ for more indices $i$. It is not difficult to see that, if $Q_{0}, \ldots, Q_{n}$ is an interpolant and $Q_{k}=\mathrm{T}$, then $\mathrm{T}, \ldots, \mathrm{T}, Q_{k+1}, \ldots, Q_{n}$ is also an interpolant. Hence the heuristic chooses an interpolant $\mathrm{T}, \ldots, \mathrm{\top}, Q_{k}, Q_{k+1}, \ldots, Q_{n}$ such that no interpolant of the form $\mathrm{T}, \ldots, \mathrm{\top}, \mathrm{\top}, Q_{k+1}^{\prime}, \ldots, Q_{n}^{\prime}$ exists, and we call such an interpolant a maximally conservative interpolant. 
The corresponding maximally conservative refinement $\varphi_{0}^{\prime}, \ldots, \varphi_{n}^{\prime}$, which is defined by $\varphi_{i}^{\prime}=\varphi_{i} \wedge Q_{i}$ $(0 \leq i \leq n)$, satisfies $\varphi_{i}^{\prime}=\varphi_{i}$ as much as possible.

Definition 6.1 (Maximally conservative refinement). Let $\varphi_{0}, \ldots, \varphi_{n}$ be a sequential representation of a partial proof that satisfies (4). Its refinement is a valid partial proof represented as $\varphi_{0}^{\prime}, \ldots, \varphi_{n}^{\prime}$ that satisfies the constraints in (5). It is $k$-conservative if $\varphi_{j}^{\prime}=\varphi_{j}$ for every $j \leq k$. It is maximally conservative if it is $k$-conservative and no $(k+1)$-conservative refinement exists.

The heuristic based on maximal conservativity introduces the following principle.

Principle 5. Use a maximally-conservative-interpolation theorem prover as the backend solver. ${ }^{6}$

We define lazy abstraction with maximally conservative interpolants, or IMPACT/MC, as a variant of McMillan's algorithm in which the interpolating theorem prover always chooses a maximally conservative one. So IMPACT/MC is an algorithm following Principles 1, 3, 4 and 5. Since Principle 5 does not specify an algorithm that computes a maximally conservative interpolant, the behavior of IMPACT/MC depends on the backend maximally-conservative-interpolation theorem prover.

The rest of this section develops algorithms for maximally conservative interpolation and compares IMPACт/MC with other software model-checking algorithms. For technical convenience, we shall focus on maximally conservative refinement instead of interpolation in the sequel.

Remark 3. The idea of maximally conservative refinement or interpolant can be found in the literature, e.g. in [Cimatti and Griggio 2012] and [Vizel and Gurfinkel 2014]. We shall compare their work with this paper in Remark 5, after establishing a connection between maximal conservativity and PDR.

\subsection{A Naïve Algorithm for Maximally Conservative Refinement}

A maximally conservative refinement is computable if binary interpolants (and thus sequential interpolants) are computable. Here we give a naïve algorithm in order to prove the computability; more sophisticated algorithms shall be introduced in the following subsections.

Algorithm 1 is a naïve algorithm that constructs a maximally conservative refinement. The naïve algorithm tries to construct a $k$-conservative refinement for $k=n, n-1, n-2, \ldots, 0$ until it finds a refinement. A $k$-conservative refinement can be computed using a sequential interpolation algorithm: letting $\varphi_{i}^{\prime}(x)=\varphi_{i}(x) \wedge Q_{i}(x)$, where $Q_{i}$ is a fresh predicate variable, it suffices to solve the constraints (6) with $\top \Rightarrow Q_{i}(x)$ for every $i \leq k$.

\subsection{Inductive Characterization of Maximally Conservative Refinement}

We show that a subproof of a maximally conservative refinement must be maximally conservative as well in a certain sense, and this property gives us an inductive characterization of maximally conservative refinements. After giving an inductive characterization of maximally conservative refinements, we develop an algorithm inspired by the characterization. We say that $\varphi_{0}^{\prime}, \ldots, \varphi_{n}^{\prime}$ is a maximally conservative refinement of $\varphi_{0}, \ldots, \varphi_{n}$ satisfying $\psi(x)$ if it is a maximally conservative refinement with respect to the constraint system ${ }^{7} S^{\prime}=\{\iota(x) \Rightarrow P(x), P(x) \wedge \tau(x, y) \Rightarrow P(y), P(x) \Rightarrow$ $\psi(x)\}$, in which the assertion $\alpha$ is replaced with $\psi$.

Lemma 6.2. Let $\left(\varphi_{i}\right)_{0 \leq i \leq n}$ be a partial proof satisfying (4). For every partial proof $\left(\psi_{0}, \ldots, \psi_{n-1}\right)$, the following conditions are equivalent:

(i) There exists $\psi_{n}$ such that $\left(\psi_{0}, \ldots, \psi_{n-1}, \psi_{n}\right)$ is a refinement of $\left(\varphi_{0}, \ldots, \varphi_{n}\right)$ satisfying $\alpha$.

\footnotetext{
${ }^{6}$ The actual implementation of IMPACт follows this principle, as we mentioned in Remark 1.

${ }^{7}$ The class of constraint systems of this form is called linear CHCs (see e.g., [Bjørner et al. 2015] for the definition).
} 


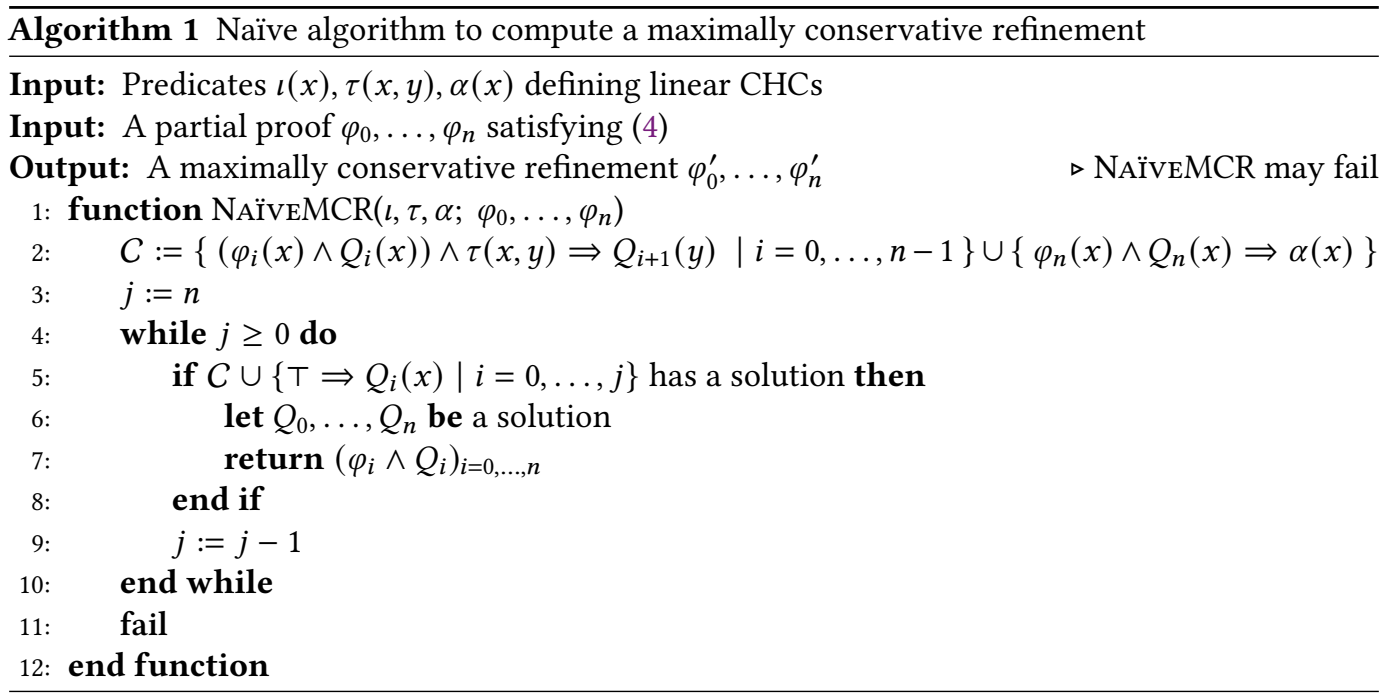

(ii) $\left(\psi_{0}, \ldots, \psi_{n-1}\right)$ is a refinement of $\left(\varphi_{0}, \ldots, \varphi_{n-1}\right)$ satisfying $\neg \exists y \cdot \tau(x, y) \wedge \neg \alpha(y)$.

Proof. Assume (1). Then $\psi_{n-1}(x) \wedge \tau(x, y) \vDash \psi_{n}(x)$ and $\psi_{n}(x) \vDash \alpha(x)$. Hence $\psi_{n-1}(x) \wedge \tau(x, y) \vDash$ $\alpha(y)$, which implies $\psi_{n-1}(x) \vDash \neg \exists y \cdot \tau(x, y) \wedge \neg \alpha(y)$.

Assume (2). Then $\psi_{n-1}(x) \vDash \neg \exists y \cdot \tau(x, y) \wedge \neg \alpha(y)$, which implies $\psi_{n-1}(x) \wedge \tau(x, y) \vDash \alpha(y)$. Since $\psi_{n-1}(x) \vDash \varphi_{n-1}(x)$ and $\varphi_{n-1}(x) \wedge \tau(x, y) \vDash \varphi_{n}(y)$ by the assumption $(4), \psi_{n-1}(x) \wedge \tau(x, y) \vDash \varphi_{n}(y)$ as well. Letting $\psi_{n}(x)$ be an interpolant of $\psi_{n-1}(x) \wedge \tau(x, y)$ and $\alpha(y) \wedge \varphi_{n}(y)$, the partial proof $\left(\psi_{0}, \ldots, \psi_{n-1}, \psi_{n}\right)$ is a refinement of $\left(\varphi_{0}, \ldots, \varphi_{n}\right)$ satisfying $\alpha$.

THEOREM 6.3. Let $\left(\varphi_{i}\right)_{0 \leq i \leq n}$ be a partial proof satisfying (4). Suppose that $\left(\varphi_{i}^{\prime}\right)_{0 \leq i \leq n}$ is a refinement of $\left(\varphi_{i}\right)_{0 \leq i \leq n}$ satisfying $\alpha$. It is maximally conservative if and only if the following conditions hold:

(a) If $\varphi_{n}(x) \vDash \alpha(x)$, then $\left(\varphi_{0}^{\prime}, \ldots, \varphi_{n}^{\prime}\right)=\left(\varphi_{0}, \ldots, \varphi_{n}\right)$.

(b) If $\varphi_{n}(x) \not \alpha(x)$, then $\left(\varphi_{0}^{\prime}, \ldots, \varphi_{n-1}^{\prime}\right)$ is a maximally conservative refinement of $\left(\varphi_{0}, \ldots, \varphi_{n-1}\right)$ satisfying $\neg \exists y \cdot \tau(x, y) \wedge \neg \alpha(y)$.

Proof. The first case is trivial. Assume that $\varphi_{n}(x) \vDash \alpha(x)$. Then there is no $n$-conservative refinement. So it suffices to show that the following conditions are equivalent for every $k<n$ :

- $\left(\varphi_{i}\right)_{0 \leq i \leq n}$ has a $k$-conservative refinement satisfying $\alpha$.

- $\left(\varphi_{i}\right)_{0 \leq i \leq n-1}$ has a $k$-conservative refinement satisfying $\neg \exists y . \tau(x, y) \wedge \neg \alpha(y)$.

This equivalence is a consequence of Lemma 6.2.

INDMCR (Algorithm 2) is an algorithm based on the characterization in Theorem 6.3. The function interpolation $(\psi(\vec{x}, \vec{y}), \vartheta(\vec{y}, \vec{z}))$ nondeterministically returns a formula from $\{\gamma(\vec{y}) \mid \psi(\vec{x}, \vec{y}) \models$ $\gamma$ and $\gamma(\vec{y}) \vDash \vartheta(\vec{y}, \vec{z})\}$, provided that $\psi(\vec{x}, \vec{y}) \vDash \vartheta(\vec{y}, \vec{z})$. Line 3 corresponds to the case (a), whereas lines 4, 5 and 7 correspond to the case (b). Hence the correctness of INDMCR is a direct consequence of Theorem 6.3. The termination can be shown by induction on $n$, since it has no loop and a recursive call takes the subproof $\left(\varphi_{0}, \ldots, \varphi_{n-1}\right)$ as its argument; note that $\varphi_{0}=\iota$ and thus, if $n=0$, exactly one of the conditions in lines 2 and 3 is true. In summary, we have the following theorem.

THEOREM 6.4. INDMCR always terminates. The set of possible return values of INDMCR coincides with the set of maximally conservative refinements. 


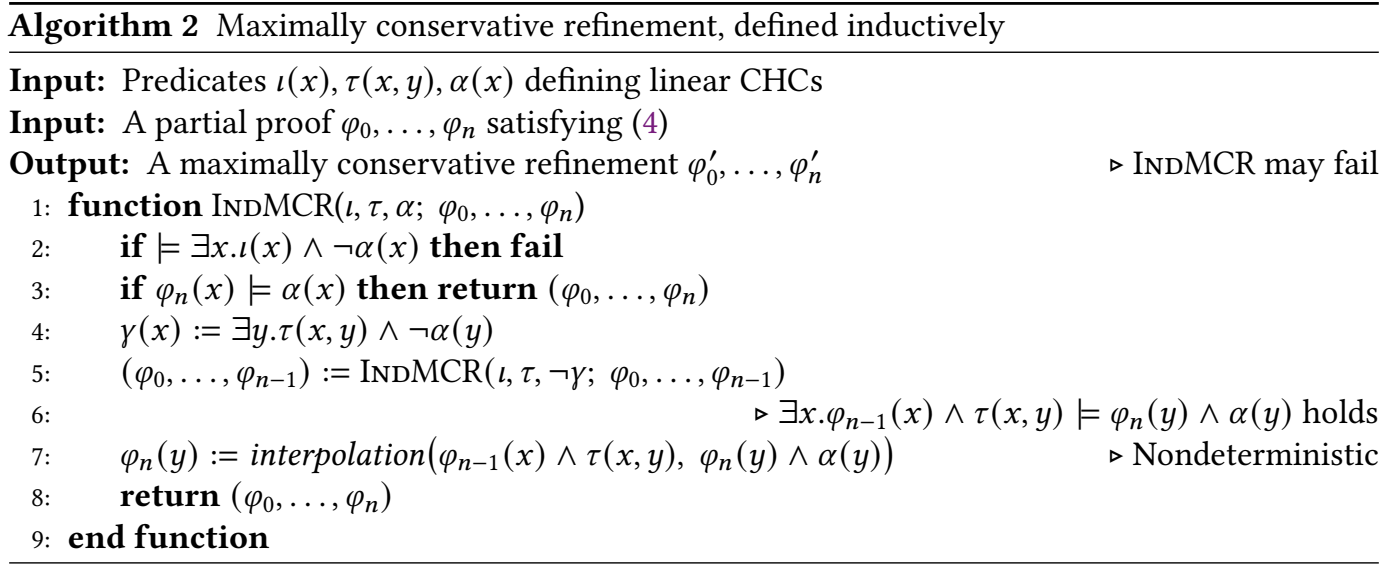

\subsection{Approximating Quantified-Formulas by Quantifier-Free Formulas}

INDMCR given in the previous subsection is defined by a simple induction on the structure of the partial proof. However it is not practical since it uses quantified formulas, which are hard to reason about. This section introduces an algorithm that does not pass a quantified formula as an argument.

Our goal is to remove the quantifier of $\exists y . \tau(x, y) \wedge \neg \alpha(y)$ in INDMCR. We appeal to the logical equivalence

$$
\exists y . \tau(x, y) \wedge \neg \alpha(y) \quad \Leftrightarrow \quad \bigvee_{c}(\tau(x, c) \wedge \neg \alpha(c))
$$

where the right-hand-side is the infinite disjunction indexed by concrete values $c$. This equivalence allows us to decompose a single complicated (i.e. quantified) formula into infinitely-many simple (i.e. quantifier-free) formulas. We reduce the computation of a maximally conservative refinement satisfying $\neg(\exists y . \tau(x, y) \wedge \neg \alpha(y))$ to iterative computation of maximally conservative refinements satisfying $\neg\left(\tau\left(x, c_{i}\right) \wedge \neg \alpha\left(c_{i}\right)\right)$ for $i=1,2,3, \ldots$, where $\left\{c_{1}, c_{2}, \ldots\right\}$ is an enumeration of values.

INDPDR (Algorithm 3) is an algorithm based on this idea. As its name suggests, it is closely related to a family of algorithms known as property-directed reachability. We shall discuss the connection in the next subsection.

The loop at lines $4-8$ is the computation of a maximally conservative refinement satisfying $\neg \exists y . \tau(x, y) \wedge \neg \alpha(y)$. If the algorithm actually computed maximally conservative refinement satisfying $\tau(x, c) \wedge \neg \alpha(c)$ for all $c$, it would never terminate. So INDPDR computes it only for relevant values. Line 5 chooses a value $M(y)$ and line 7 computes a maximally conservative refinement satisfying $\neg(\tau(x, c) \wedge \neg \alpha(c))$ where $c=M(y)$. Note that this is an over-approximation:

$$
\neg \exists y . \tau(x, y) \wedge \neg \alpha(y) \vDash \neg(\tau(x, c) \wedge \neg \alpha(c)) .
$$

If the loop terminates, then $\vDash \neg \exists x . \exists y \cdot \varphi_{n-1}(x) \wedge \tau(x, y) \wedge \neg \alpha(y)$, which implies $\varphi_{n-1}(x) \vDash$ $\neg \exists y \cdot \tau(x, y) \wedge \neg \alpha(y)$ as expected.

We prove the correctness.

LEMMA 6.5. Let $\left(\varphi_{i}\right)_{0 \leq i \leq n}$ be a partial proof and let $\alpha_{1}, \alpha_{2}$ be formulas such that $\alpha_{2}(x) \vDash \alpha_{1}(x)$. Suppose that $\left(\varphi_{i}^{\prime}\right)_{0 \leq i \leq n}$ is a maximally conservative refinement of $\left(\varphi_{i}\right)_{i}$ satisfying $\alpha_{1}$ and that $\left(\varphi_{i}^{\prime \prime}\right)_{0 \leq i \leq n}$ is a maximally conservative refinement of $\left(\varphi_{i}^{\prime}\right)_{0 \leq i \leq n}$ satisfying $\alpha_{2}$. Then $\left(\varphi_{i}^{\prime \prime}\right)_{i}$ is a maximally conservative refinement of $\left(\varphi_{i}\right)_{i}$ satisfying $\alpha_{2}$.

Proof. Assume a $k$-conservative refinement $\left(\psi_{i}\right)_{0 \leq i \leq n}$ of $\left(\varphi_{i}\right)_{i}$ satisfying $\alpha_{2}$. It suffices to show that $\left(\varphi_{i}^{\prime \prime}\right)_{i}$ is also $k$-conservative. Since $\alpha_{2}(x) \vDash \alpha_{1}(x)$, the partial proof $\left(\psi_{i}\right)_{i}$ is also a refinement of 


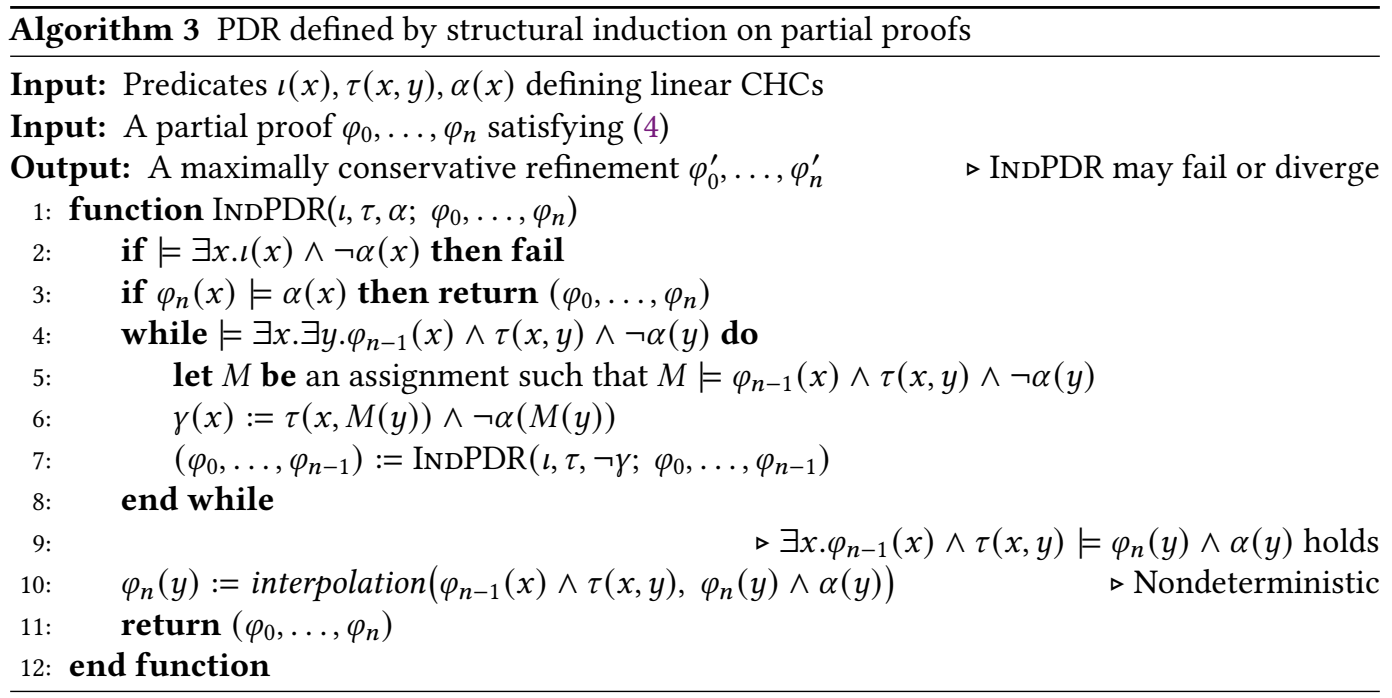

$\left(\varphi_{i}\right)_{i}$ satisfying $\alpha_{1}$. Since $\left(\varphi_{i}^{\prime}\right)_{i}$ is maximally conservative, it is $k$-conservative. Then $\left(\psi_{i} \wedge \varphi_{i}^{\prime}\right)_{0 \leq i \leq n}$ is a $k$-conservative refinement of $\left(\varphi_{i}^{\prime}\right)_{i}$ satisfying $\alpha_{1}$. By the maximal conservativity of $\left(\varphi_{i}^{\prime \prime}\right)_{i}$, it is $k$-conservative as well.

LEMMA 6.6. If INDPDR terminates, it returns a maximally conservative refinement.

Proof. Let $\left(\psi_{i}\right)_{0 \leq i \leq n}$ be the initial value of $\left(\varphi_{i}\right)_{0 \leq i \leq n}$ and $\left(\psi_{i}^{\prime}\right)_{0 \leq i \leq n}$ be the value of $\left(\varphi_{i}\right)_{i}$ at the end of the loop (or, equivalently, line 9). It suffices to show that $\left(\psi_{i}^{\prime}\right)_{0 \leq i \leq n-1}$ is a maximally conservative refinement of $\left(\psi_{i}\right)_{0 \leq i \leq n-1}$ satisfying $\neg \exists y \cdot \tau(x, y) \wedge \neg \alpha(y)$. Clearly $\psi_{n-1}^{\prime}(x) \vDash \neg \exists y \cdot \tau(x, y) \wedge \neg \alpha(y)$ since the condition in line 4 is false for the values at line 9 . The maximal conservativity can be proved by induction on the number of iterations using Lemma 6.5 since $\neg \exists y . \tau(x, y) \wedge \neg \alpha(y) \vDash$ $\neg(\tau(x, c) \wedge \neg \alpha(c))$ for every $c$.

Other properties of INDPDR shall be discussed below.

\subsection{PDR and INDPDR as Transition Systems}

Property-directed reachability (or PDR) refers to the IC3 algorithm [Bradley 2011; Een et al. 2011] and its derivatives [Beyer and Dangl 2020; Birgmeier et al. 2014; Cimatti and Griggio 2012; Cimatti et al. 2014; Hoder and Bjørner 2012; Komuravelli et al. 2014, 2013]. This section compares PDR and IMPACT/MC, in particular IMPACT/MC with INDPDR. Since PDR is formalized as an abstract transition system in [Hoder and Bjørner 2012], we give a transition system corresponding to IмРАCT/MC and compare it with the transition system in [Hoder and Bjørner 2012].

INDPDR as a transition system. An abstract transition system for INDPDR can be derived by the following argument. The starting point is operational semantics of the program INDPDR. Since INDPDR is a recursive program, a configuration in the operational semantics consists of the current assignment to variables and the call-stacks. So a configuration can be written as

$$
\left(\alpha^{\prime} ; \varphi_{0}, \ldots, \varphi_{k-1}\right) \mathbf{\square}\left(M^{k}, \alpha^{k} ; \varphi_{0}^{k}, \ldots, \varphi_{k}^{k}\right)\left(M^{k+1}, \alpha^{k+1} ; \varphi_{0}^{k+1}, \ldots, \varphi_{k+1}^{k+1}\right) \ldots\left(M^{n}, \alpha^{n} ; \varphi_{0}^{n}, \ldots, \varphi_{n}^{n}\right),
$$

where the left-hand-side of $\boldsymbol{m}$ is the current variable assignment and the right-hand-side is the callstack. An abstract transition system can be obtained by using this representation of the call-stack. 


$$
\begin{gathered}
\frac{\models \varphi_{n}(c) \wedge \neg \alpha(c)}{\left(\epsilon \| \varphi_{0} \ldots \varphi_{n}\right) \longrightarrow\left(c \| \varphi_{0} \ldots \varphi_{n}\right)} \text { (CANDIDATE) } \\
\frac{\vDash \neg l\left(c_{k}\right) \quad \models \varphi_{k-1}\left(c^{\prime}\right) \wedge \tau\left(c^{\prime}, c_{k}\right)}{\left(c_{k} \ldots c_{n} \| \varphi_{0} \ldots \varphi_{n}\right) \longrightarrow\left(c^{\prime} c_{k} \ldots c_{n} \| \varphi_{0} \ldots \varphi_{n}\right)} \text { (DECIDE) } \\
\frac{\varphi_{k-1}(x) \wedge \tau(x, y) \vDash \psi(y) \quad \psi(x) \vDash x \neq c_{k}}{\left(c_{k} \ldots c_{n} \| \varphi_{0} \ldots \varphi_{n}\right) \longrightarrow\left(c_{k+1} \ldots c_{n} \| \varphi_{0} \ldots \varphi_{k-1}\left(\varphi_{k} \wedge \psi\right) \varphi_{k+1} \ldots \varphi_{n}\right)}
\end{gathered}
$$

Fig. 5. The transition rules for INDPDR.

This representation, however, has redundancy. First forgetting $\varphi_{0}^{\ell}, \ldots, \varphi_{\ell-1}^{\ell}$ in the $\ell$-th frame $\left(M^{\ell}, \alpha^{\ell} ; \varphi_{0}^{\ell}, \ldots, \varphi_{\ell}^{\ell}\right)$ does not cause any problem, since they shall be overwritten when the control will come back to the frame. Second $\alpha^{\ell}$ is redundant: $\alpha^{n}=\alpha$ and $\alpha^{\ell}(x)=\left(\tau\left(x, M^{\ell+1}(y)\right) \wedge\right.$ $\left.\neg \alpha^{\ell+1}\left(M^{\ell+1}(y)\right)\right)$ for $\ell=n-1, \ldots, k$. Third $\alpha^{\prime}=\left(\tau\left(x, M^{k}(y)\right) \wedge \neg \alpha^{k}\left(M^{k}(y)\right)\right)$. Fourth $M^{\ell}(x)$ is not used. So we obtain a compact representation:

$$
\left(M^{k}(y) \ldots M^{n}(y) \| \varphi_{0} \ldots \varphi_{k-1} \varphi_{k}^{k} \varphi_{k+1}^{k+1} \ldots \varphi_{n}^{n}\right) .
$$

Figure 5 defines the abstract transition system corresponding to INDPDR, which is the operational semantics of INDPDR with the above representation of configurations.

The above argument shows the following proposition.

Proposition 6.7. The following conditions are equivalent.

- $\operatorname{INDPDR}\left(\iota, \tau, \alpha ; \varphi_{0}, \ldots, \varphi_{n}\right)$ may return $\left(\varphi_{0}^{\prime}, \ldots, \varphi_{n}^{\prime}\right)$.

- $\left(\epsilon \| \varphi_{0}, \ldots, \varphi_{n}\right) \longrightarrow^{*}\left(\epsilon \| \varphi_{0}^{\prime}, \ldots, \varphi_{n}^{\prime}\right)$ with $\varphi_{n}^{\prime}(x) \vDash \alpha(x)$.

Before comparing the transition system with PDR, we prove a part of the correctness of INDPDR using the transition system.

LEMMA 6.8. If $\left(\varphi_{i}^{\prime}\right)_{0 \leq i \leq n}$ is a maximally conservative refinement of $\left(\varphi_{i}\right)_{0 \leq i \leq n}$, then

$$
\left(\epsilon \| \varphi_{0} \ldots \varphi_{n}\right) \longrightarrow^{*}\left(\epsilon \| \varphi_{0}^{\prime} \ldots \varphi_{n}^{\prime}\right) .
$$

Proof. Assume that $\left(\varphi_{i}^{\prime}\right)_{0 \leq i \leq n}$ is a maximally conservative refinement of $\left(\varphi_{i}\right)_{0 \leq i \leq n}$ satisfying $\alpha$. Let $k$ be the natural number such that $\varphi_{k}^{\prime}=\varphi_{k}$ but $\varphi_{k+1}^{\prime} \neq \varphi_{k+1}$. For $i=k+2, \ldots, n$, we define $\psi_{i}$ by

$$
\varphi_{k+1} \rightsquigarrow \psi_{k+2} \rightsquigarrow \psi_{k+3} \rightsquigarrow \cdots \rightsquigarrow \psi_{n}
$$

where $\rightsquigarrow$ is the precise symbolic computation. Then $\left(\varphi_{0}, \ldots, \varphi_{k+1}, \psi_{k+2}, \ldots, \psi_{n}\right)$ satisfies the requirements for refinement except for $\psi_{n}(x) \vDash \alpha(x)$. By the maximal conservativity of $\left(\varphi_{i}^{\prime}\right)_{i}$, we know that $\left(\varphi_{0}, \ldots, \varphi_{k+1}, \psi_{k+2}, \ldots, \psi_{n}\right)$ is not a refinement satisfying $\alpha$, and thus $\models \psi_{n}\left(c_{n}\right) \wedge \neg \alpha\left(c_{n}\right)$ for some $c_{n}$. Hence we have a sequence $\left(c_{k+1}, \ldots, c_{n}\right)$ of values such that $\models \varphi_{k+1}\left(c_{k+1}\right)$ and $\models \tau\left(c_{i}, c_{i+1}\right)$ for every $i=k+1, \ldots, n-1$. So we have the following transition sequence:

$$
\begin{aligned}
& \left(\epsilon \| \varphi_{0} \ldots \varphi_{n}\right) \longrightarrow^{*}\left(c_{k+1} \ldots c_{n} \| \varphi_{0} \ldots \varphi_{n}\right) \longrightarrow\left(c_{k+2} \ldots c_{n} \| \varphi_{0} \ldots \varphi_{k} \varphi_{k+1}^{\prime} \varphi_{k+2} \ldots \varphi_{n}\right) \\
& \longrightarrow\left(c_{k+3} \ldots c_{n} \| \varphi_{0} \ldots \varphi_{k} \varphi_{k+1}^{\prime} \varphi_{k+2}^{\prime} \varphi_{k+3} \ldots \varphi_{n}\right) \longrightarrow\left(\epsilon \| \varphi_{0} \ldots \varphi_{k} \varphi_{k+1}^{\prime} \varphi_{k+2}^{\prime} \cdots \varphi_{n}^{\prime}\right) .
\end{aligned}
$$

COROLLARY 6.9. The set of possible return values of INDPDR coincides with the set of all maximally conservative refinements. 


$$
\begin{gathered}
\frac{\vDash F_{n}(c) \wedge \neg \alpha(c)}{\left(\epsilon \| F_{0} \ldots F_{n}\right) \longrightarrow_{\mathrm{PDR}}\left(c \| F_{0} \ldots F_{n}\right)}(\text { CANDIDATE/sPDR) } \\
\frac{\vDash F_{k-1}\left(c^{\prime}\right) \wedge \tau\left(c^{\prime}, c_{k}\right)}{\left(c_{k} \ldots c_{n} \| F_{0} \ldots F_{n}\right) \longrightarrow_{\mathrm{PDR}}\left(c^{\prime} c_{k} \ldots c_{n} \| F_{0} \ldots F_{n}\right)}(\mathrm{DECIDE} / \mathrm{sPDR})
\end{gathered}
$$

$\frac{\left.\iota(y) \vee\left(\exists x . F_{k-1}(x) \wedge \tau(x, y)\right)\right) \vDash \psi(y) \quad F_{k-1}(x) \vDash \psi(x) \quad \psi(x) \vDash x \neq c_{k}}{\left(c_{k} \ldots c_{n} \| F_{0} \ldots F_{n}\right) \longrightarrow{ }_{\mathrm{PDR}}\left(c_{k+1} \ldots c_{n} \| F_{0} \ldots F_{k-1}\left(F_{k} \wedge \psi\right) F_{k+1} \ldots F_{n}\right)}$ (CONFLICT/SPDR)

Fig. 6. A simplified version of the transition rules for PDR in [Hoder and Bjørner 2012]; see Remark 4 for the differences. We omit (UNFOLD), (INITIALIZE), (VALID) and (MODEL), as these rules are not a part of the refinement/interpolation process. These rules corresponds the content described in Definition 6.10.

Definition of PDR. We review PDR following Hoder and Bjørner [Hoder and Bjørner 2012]. Here we introduce a simplified version, which we call simplified PDR $(s P D R)$; see Remark 4 for the differences. A configuration is of the form $\left(c_{k} \ldots c_{n} \| F_{0} F_{1} \ldots F_{n}\right)$, consisting of two sequences of (candidate) counterexamples $c_{i} \in \mathcal{S}$ and frames $F_{i},{ }^{8}$ which are formulas denoting unary predicates. We call $c_{i}$ (resp. $F_{i}$ ) the counterexample (resp. the frame) at level $i$. Note that counterexamples are associated to only higher levels and that the counterexample sequence can be empty $\epsilon$. Intuitively a frame $F_{i}$ at level $i$ denotes an over-approximation of states of distance $\leq i$ and $c_{i}$ together with $c_{i+1} \ldots c_{n}$ is a witness of unsafety of $F_{i}$ with bound $(n-i)$, i.e. $F_{i}\left(c_{i}\right)$ and $\bigwedge_{j=i}^{n-1} \tau\left(c_{j}, c_{j+1}\right) \wedge \neg \alpha\left(c_{n}\right)$ Formally each configuration $\left(c_{k} \ldots c_{n} \| F_{0} \ldots F_{n}\right)$ must satisfy (a) $F_{0}=\iota$, (b) $F_{i}(x) \vee \mathcal{F}\left(F_{i}\right)(x) \vDash$ $F_{i+1}(x)$ for every $0 \leq i \leq n,{ }^{9}$ (c) $F_{i}(x) \vDash \alpha(x)$ for every $0 \leq i<n$, (d) $\vDash F_{i}\left(c_{i}\right)$ for every $k \leq i \leq n$, and $(\mathrm{e}) \vDash \bigwedge_{i=k}^{n-1} \tau\left(c_{i}, c_{i+1}\right) \wedge \neg \alpha\left(c_{n}\right)$.

The transition rules are given in Figure 6. The rule (UNFOLD) is applicable when $F_{0}=\iota$ has been confirmed safe with bound $n$. This rule starts to check if $\iota$ is safe with bound $(n+1)$, by extending the frame by T. (CANDIDATE) is applicable when a state $c_{n}$ satisfying $F_{n}$ violates the assertion $\alpha$ and starts to check if the bad state $c_{n}$ is $n$-step reachable from $\iota$. The candidate counterexample is propagated backward by (DECIDE) rule. If (DECIDE) is not applicable, the candidate is spurious, i.e. it was introduced by the approximation at this level. Then (CоNFLICT) refines the approximation, strengthening $F_{k}$ so that it does not contain the spurious counterexample $c_{k}$ (i.e. $\psi(x) \vDash x \neq c_{k}$ ).

Definition 6.10 (Simplified PDR). The algorithm sPDR is defined as follows. It starts from the initial configuration $(\epsilon \| l)$. The algorithm iteratively rewrites the configuration. If it reaches ( $\left.\epsilon \| F_{0} \ldots F_{n}\right)$ with $F_{\ell}(x) \vDash F_{\ell-1}(x)$ for some $\ell$, then the program is safe and an invariant is $F_{\ell}$. If it reaches $\left(c_{0} \ldots c_{n} \| F_{0} \ldots F_{n}\right)$ with a counterexample at level 0 , the program is unsafe. If it reaches $\left(\epsilon \| F_{0} \ldots F_{n}\right)$ with $F_{n}(x) \models \alpha$, then it moves to $\left.\epsilon \| F_{0} \ldots F_{n} \top\right)$.

sPDR vs. IMPACT/MC with INDPDR. sPDR in Figure 6 is essentially the same as IMPACT/MC with INDPDR. Consider the relation $\approx$ between configurations of INDPDR and of SPDR defined by

$$
\left(c_{k} \ldots c_{n} \| \varphi_{0} \ldots \varphi_{n}\right) \approx\left(c_{k} \ldots c_{n} \| F_{0} \ldots F_{n}\right) \quad \text { iff } \quad \vDash F_{j}(x) \Leftrightarrow \bigvee_{0 \leq i \leq j} \varphi_{i}(x) \text { for every } 0 \leq j \leq n
$$

This relation $\approx$ is a bisimilation.

Theorem 6.11. Suppose that $(\vec{c} \| \vec{\varphi}) \approx(\vec{c} \| \vec{F})$. If $(\vec{c} \| \vec{\varphi}) \longrightarrow\left(\vec{c}^{\prime} \| \vec{\varphi}^{\prime}\right)$, then $(\vec{c} \| \vec{F}) \longrightarrow_{P D R}$ $\left(\vec{c}^{\prime} \| \vec{F}^{\prime}\right)$ and $\left(\vec{c}^{\prime} \| \vec{\varphi}^{\prime}\right) \approx\left(\vec{c}^{\prime}, \| \vec{F}^{\prime}\right)$ for some $\left(\vec{c}^{\prime} \| \vec{F}^{\prime}\right)$. Conversely, if $(\vec{c} \| \vec{F}) \longrightarrow_{P D R}\left(\vec{c}^{\prime} \| \vec{F}^{\prime}\right)$, then $(\vec{c} \| \vec{\varphi}) \longrightarrow\left(\vec{c}^{\prime} \| \vec{\varphi}^{\prime}\right)$ and $\left(\vec{c}^{\prime} \| \vec{\varphi}^{\prime}\right) \approx\left(\vec{c}^{\prime} \| \vec{F}^{\prime}\right)$ for some $\left(\vec{c}^{\prime} \| \vec{\varphi}^{\prime}\right)$.

\footnotetext{
${ }^{8}$ We do not use metavariables $\varphi$ and $\psi$ for frames, because a frame does not precisely correspond to a cut-formula. See a discussion below.

${ }^{9} \mathcal{F}$ is the predicate transformer given by $\mathcal{F}(F)(y):=\iota(y) \vee(\exists x . F(x) \wedge \tau(x, y))$.
} 
Proof. This is intuitively clear as each rule has a counterpart, but we need some care to establish the precise correspondence.

(CANDIDATE/sPDR) can be simulated by (CANDIDATE). To see this, we have to check that $\models$ $F_{n}(c) \wedge \neg \alpha(c)$ implies $\vDash \varphi_{n}(c) \wedge \neg \alpha(c)$. This follows from the assumption $F_{n}(x) \Leftrightarrow \bigvee_{0 \leq i \leq n} \varphi_{i}(x)$ and $\varphi_{i}(x) \vDash \alpha(x)$ for $i<n$. The conserve is trivial because $\varphi_{n}(x) \vDash F_{n}(x)$.

The correspondence of (DECIDE/sPDR) and (DECIDE) can be show similarly.

It is easy to see that (CONFLICT) with interpolation $\psi$ coincides with (CONFLICT/SPDR) with the same interpolation $\psi$.

Remark 4. sPDR defined by Figure 6 differs from that by Hoder and Bjørner [Hoder and Bjørner 2012], which has an additional rule (INDUCTION/PDR) and a slightly weaker condition for the interpolants as in (CONFLICT/PDR) below:

$$
\begin{gathered}
\frac{\left.\iota(y) \vee\left(\exists x .\left(F_{\ell-1}(x) \wedge \psi(x)\right) \wedge \tau(x, y)\right)\right) \vDash \psi(y)}{\left(c_{k} \ldots c_{n} \| F_{0} \ldots F_{n}\right) \longrightarrow \longrightarrow_{\mathrm{PDR}}\left(c_{k} \ldots c_{n} \|\left(F_{0} \wedge \psi\right) \ldots\left(F_{\ell} \wedge \psi\right) F_{\ell+1} \ldots F_{n}\right)} \text { (INDUCTION/PDR) } \\
\frac{\left.\iota(y) \vee\left(\exists x . F_{k-1}(x) \wedge \tau(x, y)\right)\right) \vDash \psi(y) \quad \psi(x) \vDash x \neq c_{k}}{\left(c_{k} \ldots c_{n} \| F_{0} \ldots F_{n}\right) \longrightarrow_{\mathrm{PDR}}\left(c_{k+1} \ldots c_{n} \|\left(F_{0} \wedge \psi\right) \ldots\left(F_{k} \wedge \psi\right) F_{k+1} \ldots F_{n}\right)} \text { (ConFLICT/PDR) }
\end{gathered}
$$

Notice that, in order to keep the monotonicity $F_{i}(x) \vDash F_{i+1}(x)$, we have to conjoin $\psi$ to $F_{j}$ $(0 \leq j<k)$ as well. This version of PDR coincides with an extension of INDPDR by the following rule corresponding to (INDUCTION/PDR):

$$
\frac{\iota(y) \vDash \psi(y) \quad \exists x .\left(\varphi_{i}(x) \wedge \psi(x)\right) \wedge \tau(x, y) \vDash \psi(y) \text { for } i=0,1, \ldots, \ell-1}{\left(c_{k} \ldots c_{n} \| \varphi_{0} \ldots \varphi_{n}\right) \longrightarrow\left(c_{k} \ldots c_{n} \|\left(\varphi_{0} \wedge \psi\right) \ldots\left(\varphi_{\ell} \wedge \psi\right) \varphi_{\ell+1} \ldots \varphi_{n}\right)} \text { (IndUCTION) }
$$

Remark 5. A similarity between PDR and McMillan's lazy abstraction with interpolants was already recognized as in [Cimatti and Griggio 2012; Een et al. 2011]. They pointed out that a multi-step transition $\left(\epsilon \| \varphi_{0} \ldots \varphi_{n-1} \top\right) \longrightarrow^{*}\left(\epsilon \| \varphi_{0}^{\prime} \ldots \varphi_{n-1}^{\prime} \varphi_{n}^{\prime}\right)$ (with $\left.\varphi_{n}^{\prime}(x) \vDash \alpha(x)\right)$ is indeed a calculation of an interpolant. Hence PDR "can be seen as an instance of the lazy abstraction with interpolants algorithm of [McMillan 2006], in which however interpolants are constructed in a very different way" [Cimatti and Griggio 2012]. Here we further investigate the connection, giving a complete characterization of interpolants given by PDR (Theorem 6.11). To the best of our knowledge, this is the first declarative characterization of interpolants computed by PDR.

The maximal conservativity heuristic and a naïve algorithm for computing a maximally conservative interpolant (Algorithm 1) can be found in [Vizel and Gurfinkel 2014] (called min-suffix heuristic), which aims to combine the interpolation-based approach and PDR. This paper differs from the work in the following points. First this paper formally proves that IMPACT with the maximal conservativity heuristic is essentially the same as PDR, whereas [Vizel and Gurfinkel 2014] regards it as a heuristic for interpolation-based model-checkers and does not establish its formal connection to PDR. Second, this paper also analyzes other algorithms from the view point of maximal conservativity (see Section 7).

\subsection{Application: Making PDR refutationally complete by model-based projection}

In contrast to the naïve algorithm NAÏvEMCR (Algorithm 1), which always terminates, INDPDR (Algorithm 3) may diverge. Therefore IMPACT/MC with INDPDR does not have refutational completeness because, even if an input program (or an input linear $\mathrm{CHC}$ system) has an error path of length $n$, the backend solver INDPDR may diverge during the construction of a proof of the safety of the program within $(n-1)$ steps. 
This subsection develops a variant of PDR with refutational completeness, in order to demonstrate the usefulness of our logical analysis of PDR. To the best of our knowledge, the algorithm developed here is the first PDR for software model-checking with refutational completeness. ${ }^{10}$

INDPDR may diverge because the loop starting from line 4 in Algorithm 3 may continue indefinitely. Let

$$
\text { BadReachable }_{n}:=\left\{c \mid \vDash \exists x \cdot \varphi_{n-1}(x) \wedge \tau(x, c) \wedge \neg \alpha(c)\right\}
$$

be the set of bad states that is reachable from $\varphi_{n-1}$ in one step. In each loop, INDPDR chooses a state $M(y)=c \in$ BadRechable $_{n}$ and tries to refine the immediate subproof $\varphi_{0}, \ldots, \varphi_{n-1}$ to ensure that $c$ is in fact unreachable. BadReachable $e_{n}$ decreases in each iteration, and the loop terminates when BadReachable $_{n}$ becomes empty. In the worst case, each loop removes only one element, namely the chosen element $c$, from BadReachable ${ }_{n}$. Hence, if BadReachable ${ }_{n}$ is infinite, infinite iteration of the loop may be required to make BadReachable $e_{n}$ empty. This divergent behavior has been observed in [Komuravelli et al. 2014] for a variant of PDR known as GPDR [Hoder and Bjørner 2012].

From a logical point of view, this phenomenon can be explained as follows. Recall that the aim of INDPDR is to remove the quantification from $\exists y . \tau(x, y) \wedge \neg \alpha(y)$ in INDMCR (Algorithm 2). The idea is to replace the variable $y$ with a concrete value, and the point is the logical equivalence

$$
\exists y \cdot \tau(x, y) \wedge \neg \alpha(y) \Leftrightarrow \quad \bigvee_{c}(\tau(x, c) \wedge \neg \alpha(c))
$$

where the right-hand-side is the infinite disjunction. This allows us to decompose a quantified formula $\exists y . \tau(x, y) \wedge \neg \alpha(y)$ into an infinite collection of quantifier-free formulas $\tau(x, c) \wedge \neg \alpha(c)$. So the essence of INDPDR is a decomposition of a single hard query into infinitely many tractable queries, and the infinity causes divergence in the worst case.

Model-based projection [Komuravelli et al. 2014] was introduced to overcome the problem.

Definition 6.12 (Model-based projection [Komuravelli et al. 2014]). Let $\varphi(\vec{x}, \vec{y})$ be a quantifier-free formula. A function $\operatorname{MBP}(\varphi,\{\vec{y}\},-)$ from models $M$ of $\varphi$ (i.e. assignments for $\vec{x}$ and $\vec{y}$ such that $M \vDash \varphi)$ to quantifier-free formulas $\psi_{M}=\operatorname{MBP}(\varphi,\{\vec{y}\}, M)$ with $\mathrm{fv}\left(\psi_{M}\right) \subseteq\{\vec{x}\}$ is a model-based projection for $\varphi$ with respect to $\vec{y}$ if it satisfies

$$
\exists \vec{y} . \varphi(\vec{x}, \vec{y}) \Leftrightarrow \bigvee_{M \models \varphi} \psi_{M}(\vec{x}) \quad \text { and } \quad M \vDash \psi_{M}(\vec{x}) \text { for every model } M
$$

and furthermore the image $\left\{\psi_{M} \mid M \models \varphi\right\}$ is finite. We assume that a model-based projection exists for every pair of $\varphi$ and $\vec{y}$ in the constraint language.

The condition (9) is an abstraction of the property of the mapping $\operatorname{Subst}(\varphi(\vec{x}, \vec{y}),\{\vec{y}\}, M):=$ $\varphi(\vec{x}, M(\vec{y}))$, so model-based projection is a generalization of the decomponition in Equation 8. A significant difference is the finiteness of the image: the disjunction $\bigvee_{M \models \varphi} \psi_{M}(\vec{x})$ is actually a finite disjunction.

By using our inductive description of PDR, it is surprisingly easy to incorporate model-based projection into INDPDR: we simply replace the substitution $\tau(x, M(y)) \wedge \neg \alpha(M(y))$ of the value $M(y)$ for $y$ (line 6 in Algorithm 3) with model-based projection $M B P(\tau(x, y) \wedge \neg \alpha(y),\{y\}, M)$. The resulting algorithm, which we call INDPDR/MBP, is shown in Algorithm 4.

TheOREM 6.13. INDPDR/MBP always terminates.

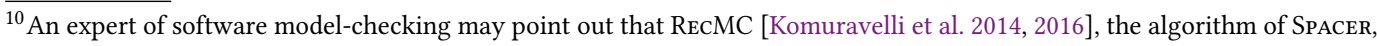
is a variant of PDR with refutational completeness. Although a proof sketch of refutational completeness of RECMC is given in [Komuravelli et al. 2016], we found a counterexample, which shall be discussed at the end of this subsection.
} 


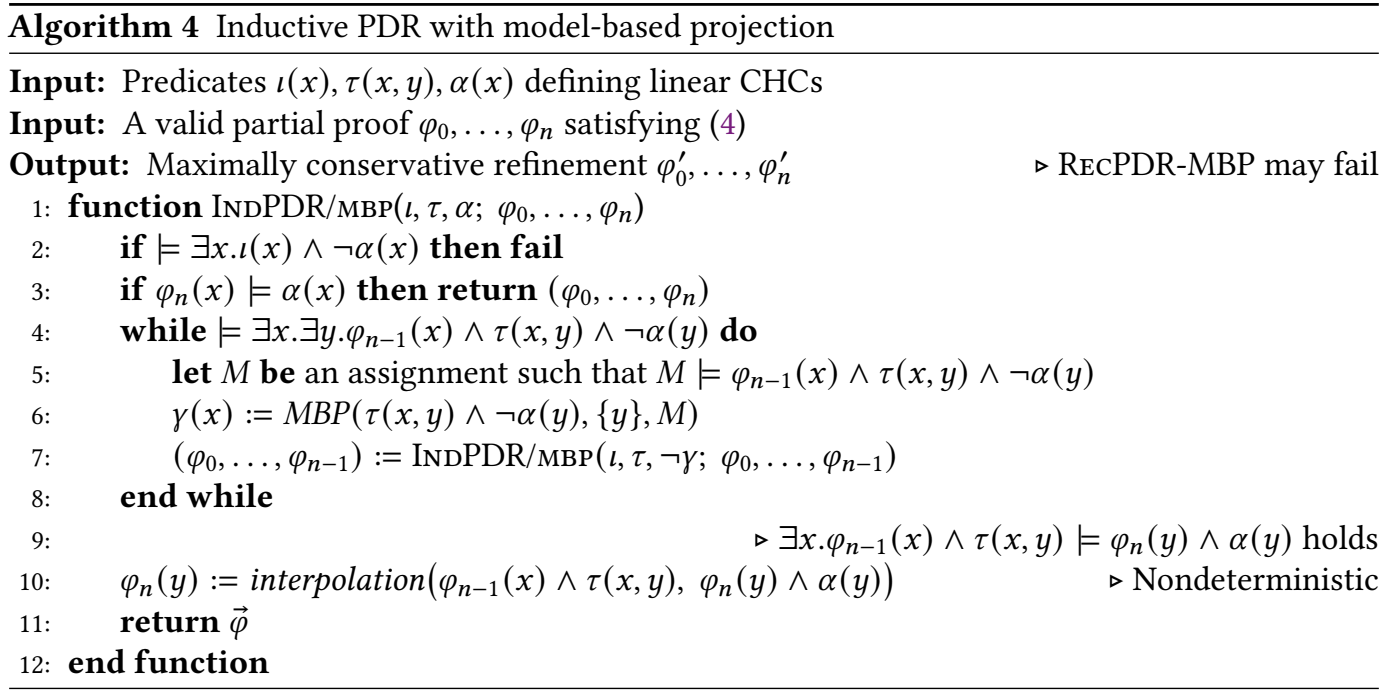

$$
\begin{gathered}
\overline{\left(\epsilon \| \varphi_{0} \ldots \varphi_{n}\right) \longrightarrow\left(\alpha \| \varphi_{0} \ldots \varphi_{n}\right)}(\text { CAndidate/MBP }) \\
\vDash \neg \exists x . l(x) \wedge \gamma_{k}(x) \quad M \vDash \varphi_{k-1}(x) \wedge \tau(x, y) \wedge \gamma_{k}(y) \\
\frac{\gamma_{k-1}(x)=M B P\left(\tau(x, y) \wedge \gamma_{k}(y),\{y\}, M\right)}{\left(\gamma_{k} \ldots \gamma_{n} \| \varphi_{0} \ldots \varphi_{n}\right) \longrightarrow\left(\gamma_{k-1} \gamma_{k} \ldots \gamma_{n} \| \varphi_{0} \ldots \varphi_{n}\right)}(\text { DeCIDE/MBP }) \\
\frac{\varphi_{k-1}(x) \wedge \tau(x, y) \vDash \psi(y) \quad \psi(x) \vDash \neg \gamma_{k}(x)}{\left(\gamma_{k} \ldots \gamma_{n} \| \varphi_{0} \ldots \varphi_{n}\right) \longrightarrow\left(\gamma_{k+1} \ldots \gamma_{n} \| \varphi_{0} \ldots \varphi_{k-1}\left(\varphi_{k} \wedge \psi\right) \varphi_{k+1} \ldots \varphi_{n}\right)} \text { (CONFLICT/MBP) }
\end{gathered}
$$

Fig. 7. The transition rules for PDR with model-based projection.

Proof. We prove the claim by induction on $n$. If $n=0$, then $\varphi_{0}=\varphi_{n}=\iota$ by (4). Since

$$
\neg(\exists x . \iota(x) \wedge \neg \alpha(x)) \quad \text { iff } \quad \forall x . \iota(x) \Rightarrow \alpha(x) \quad \text { iff } \quad \forall x . \varphi_{n}(x) \Rightarrow \alpha(x),
$$

RECPDR-MBP fails in line 2 or returns a value in line 3.

Assume that $n>0$. By the induction hypothesis, the recursive call in line 7 terminates. It suffices to show the termination of the loop starting from line 4.

Assume for contradiction that the loop continues indefinitely. For each $i=1,2, \ldots$, let $M_{i}$ and $\gamma_{i}$ be $M$ and $\alpha^{\prime}$ in the $i$-th loop and $\psi_{i}$ be $\varphi_{n-1}$ at the end of the $i$-th loop. By construction, we have

$$
M_{i} \vDash \psi_{i-1}(x) \wedge \tau(x, y) \wedge \neg \alpha(x) \quad \text { and } \quad M_{i} \vDash \gamma_{i}(x)
$$

for each $i=1,2, \ldots$. By the definition of refinement,

$$
\psi_{i}(x) \vDash \neg \gamma_{i}(x) \text { and } \quad \psi_{i}(x) \vDash \psi_{i-1}(x)
$$

where $\psi_{0}$ is $\varphi_{n-1}$ before the loop. Suppose $i<j$. Since $M_{j} \vDash \psi_{j-1}(x)$ and $\forall k .\left[\psi_{k}(x) \vDash \psi_{k-1}(x)\right]$, we have $M_{j} \vDash \psi_{i}(x)$. Hence $M_{j} \vDash \neg \gamma_{i}(x)$ since $\psi_{i}(x) \vDash \neg \gamma_{i}(x)$. As $M_{i} \vDash \gamma_{i}(x)$, we have $\gamma_{i}(x) \neq \gamma_{j}(x)$ if $i<j$. This contradicts the finiteness assumption of MBP since $\gamma_{i}(x)=\operatorname{MBP}\left(\tau(x, y) \wedge \neg \alpha(y),\{y\}, M_{i}\right)$ and the formula $\tau(x, y) \wedge \neg \alpha(y)$ is a constant in the loop.

It is not difficult to give an abstract transition system corresponding to INDPDR/MBP (Algorithm 4). It is shown in Figure 7. 
Subtlety of model-based projection. Algorithms using MBP should be carefully designed. For example, let INDPDR/MBP' be the algorithm obtained by replacing line 6 of INDPDR/MBP with

$$
\gamma(x):=\operatorname{MBP}\left(\varphi_{n-1}(x) \wedge \tau(x, y) \wedge \neg \alpha(y),\{y\}, M\right) .
$$

This small change breaks the termination property of the algorithm. This is because $\varphi_{k-1}(x)$ is changed in each iteration of the loop; hence a different iteration calculates model-based projection of different formulas. In this case, the finiteness property of model-based projection does not help, since the finiteness is ensured only if model-based projection is applied to a single formula.

We give a small toy example to prove that INDPDR/MBP' may diverge. Let us consider the following unsatisfiable linear $\mathrm{CHC}$ system

$$
S=\{x=0 \Rightarrow P(x), P(x) \wedge(y=x+1 \vee y=1-2 x) \Rightarrow P(y), P(x) \Rightarrow x \leq 2\},
$$

so $\iota(x)=(x=0), \tau(x, y)=(y=x+1 \vee y=1-2 x)$ and $\alpha(x)=(x \leq 2)$. Let $\varphi_{0}=\imath, \varphi_{1}(x)=(x \leq 1)$ and $\varphi_{2}(x)=\mathrm{T}$. Then $\left(\varphi_{0}, \varphi_{1}, \varphi_{2}\right)$ has a refinement, e.g. $(x=0, x=1, x \leq 2)$. Let $M B P$ be an arbitrary model-based projection method. We define $M B P^{\prime}$ by

$$
M B P^{\prime}(\psi(x, y),\{y\}, M):= \begin{cases}x=m & \text { if } M(x)=m=\max \{n<0 \mid \vDash \exists y . \psi(n, y)\} \\ M B P(\psi(x, y),\{y\}, M) & \text { otherwise. }\end{cases}
$$

We also assume that

$$
\text { interpolation }(\psi(x, y), \vartheta(y)):=\vartheta(y)
$$

Then INDPDR/MBP $\left(\iota, \tau, \alpha ; \varphi_{0}, \varphi_{1}, \varphi_{2}\right)$ diverges, provided that the model $M_{i}$ in the $i$-th iteration, $i=1,2, \ldots$, of the loop is $\left(M_{i}(x), M_{i}(y)\right)=(-i, 1+2 i)$. Then $\varphi_{1}$ at the end of $i$-th iteration is $(x \leq 1 \wedge x \neq-1 \wedge \cdots \wedge x \neq-i)$, and the loop never terminates.

RECMC [Komuravelli et al. 2014, 2016], which is the algorithm used in the state-of-the-art software model-checker SPACER, has the same problem as INDPDR/MBP'. It applies model-based projection to a formula containing the current over- and/or under-approximations, which vary over time; see (QUERY) rule in [Komuravelli et al. 2016, Fig. 7] (where the existential quantifier in the definition of $\psi$ shall be replaced with mobel-based projection in a later section). This observation applies to other formalizations of SPACER, e.g. [Komuravelli et al. 2015] (that actually behaves slightly differently).

We give a concrete counterexample. Let $S^{\prime}$ be a non-linear $\mathrm{CHC}$ system, given by

$$
\{\iota(x) \Rightarrow Q(0, x), \quad \top \Rightarrow Q(1, x), \quad Q(0, x) \wedge Q(1, x) \wedge \tau(x, y) \Rightarrow Q(0, y), \quad Q(0, x) \Rightarrow \alpha(x)\} .
$$

Since $Q(1, x)=\top$, one can remove $Q(1, x)$ from $S^{\prime}$ by substituting $\mathrm{T}$ for it. The resulting constraint set is essentially the same as $S$, obtained by replacing $P(x)$ in $S$ with $Q(0, x)$. A badly-behaved model-based projection $M B P^{\prime \prime}$ is similar to $M B P^{\prime}$ : it basically behaves as $M B P$ but returns $x=m$ if $M(x)=m$ is either the maximum or minimum in $\{n<0 \mid \vDash \exists y . \psi(n, y)\}$.

This divergent behavior is notable because a journal paper [Komuravelli et al. 2016] proves that RECMC always terminates without assuming any assumptions on the model-based projection process and the interpolating theorem prover. This shows the nontriviality and subtlety of termination proofs of algorithms with model-based projection.

\section{MAXIMAL CONSERVATIVITY FOR INFINITE GAME SOLVING}

The characterization of PDR by maximal conservativity reveals an unexpected connection between PDR and an efficient procedure for game solving [Farzan and Kincaid 2017].

Farzan and Kincaid [Farzan and Kincaid 2017] proposed a procedure for game solving over infinite graphs of which rules are defined by linear arithmetic. Assume that a state is represented by a tuple $\vec{s}=\left(s_{1}, \ldots, s_{n}\right)$ of data of the logic, as well as actions $\vec{a}_{0}$ and $\vec{a}_{1}$ of Players 0 and 1 , 
respectively. Players alternatively choose actions, starting from Player 0 . The transition of the game is a partial function represented by $\tau: \tau\left(\vec{s}, \vec{a}_{0}, \vec{a}_{1}, \vec{t}\right)$ is true if and only if $\vec{t}$ is the state after playing $\vec{a}_{0}$ and then $\vec{a}_{1}$. Player 0 wins the game if the game does not get stuck. We are interested in whether Player 0 wins the game.

Their approach is fairly naturally understood as a cyclic proof search, although their paper [Farzan and Kincaid 2017] does not mention the connection. The winning region of Player 0 is characterized by the greatest solution of

$$
W(\vec{s}) \Leftrightarrow \exists \vec{a}_{0} \cdot \forall \vec{a}_{1} \cdot \exists \vec{t} . \tau\left(\vec{s}, \vec{a}_{0}, \vec{a}_{1}, \vec{t}\right) \wedge W(\vec{t}) .
$$

The definition says that $\vec{s}$ is winning for Player 0 if there exists an appropriate action $\vec{a}_{0}$ of Player 0 such that, for every action $\vec{a}_{1}$ of Player 1 , the next state $\vec{t}$ exists and it is a winning position of Player 0 . So the game solving is reduced to the validity of $\iota(\vec{s}) \vdash(v W)(\vec{s})$.

Their procedure can be described as a proof search strategy for sequents of the form $\varphi(\vec{s})+$ $(v W)(\vec{s})$. Application of $(v-\mathrm{R})$ yields a partial proof with an open sequent $\varphi(\vec{s}) \vdash \delta[v W](\vec{s})$, where $\delta$ is the right-hand-side of Equation 10. A significant difference from software model-checking is found here: since $\delta$ contains quantifiers, it is not easy to transform this sequent into $\psi(\vec{t}) \vdash(v W)(\vec{t})$. Their idea is to use a proof of a fixed-point-free sequent $\varphi(\vec{s}) \vdash \delta[\top](\vec{s})$, from which one can easily obtain a partial proof by replacing T with $(v W)$. To (dis)prove the sequent $\varphi(\vec{s}) \vdash \delta[T](\vec{s})$, they used an external solver, which itself is developed in the first half of their paper [Farzan and Kincaid 2017] using [Farzan and Kincaid 2016].

What is notable is a way to fix the partial proof when $\varphi(\vec{s}) \vdash \delta[T](\vec{s})$ is false. Recall that $\varphi(\vec{s}) \vdash(v W)(\vec{s})$ is an open sequent of the current partial proof, which is a leaf. Their procedure tries to fix the partial proof, by refining a subtree containing this open sequent. Their procedure first seeks the nearest ancestor of the open sequent $\varphi(\vec{s}) \vdash(v W)(\vec{s})$ that uses $(v-\mathrm{R})$ rule in the current partial proof. Let $\varphi^{\prime}\left(\vec{s}^{\prime}\right) \vdash \delta[v W]\left(\vec{s}^{\prime}\right)$ be its premise. Then the procedure check the validity of $\varphi^{\prime}\left(\vec{s}^{\prime}\right) \vdash \delta[\delta[T]]\left(\vec{s}^{\prime}\right)$, the two-folded expansion of $v W$. If the sequent is valid, it replaces the subtree above the ancestor by using the proof; if the sequent is invalid, it seeks the next ancestor and tries to fix the subtree above it. This is the maximally conservative policy, and their procedure for game solving belongs to the PDR family in this sense.

We conclude with two consequences. First the notion of maximal conservativity is a useful characterization of PDR that significantly extends the scope of PDR. Second the maximal conservativity would be useful for implementing an efficient prover: at least, it works well for sequents corresponding to game solving to some extent, as confirmed by the experiments in [Farzan and Kincaid 2017].

\section{RELATED WORK}

We have demonstrated that not only well-known software model checking algorithms, including symbolic execution, bounded model checking [Biere et al. 1999], predicate abstraction [Ball et al 2001], lazy abstraction [Henzinger et al. 2004, 2002; McMillan 2006], and PDR [Cimatti and Griggio 2012; Hoder and Bjørner 2012], but also an efficient game solving algorithm [Farzan and Kincaid 2017] can be seen as cyclic-proof search. This section discusses other related proof search and software model-checking algorithms.

\subsection{Proof Sarch in Cyclic and Martin-Löf-Style Proof Systems}

Proof search in cyclic [Brotherston and Simpson 2011; Sprenger and Dam 2003] and Martin-Löfstyle [Martin-Löf 1971] proof systems finds wide applications in safety/liveness program verification and entailment checking in first-order and separation logics with inductive predicates. 
Program verification. Brotherston et al. [Brotherston et al. 2008, 2012; Tellez and Brotherston 2020] gave cyclic proof systems for Hoare logic (with the separation logic as the underlying assertion language). They mentioned a certain part of a proof search corresponds to symbolic execution. However, they did not establish a connection to modern software model-checkers, of which the main challenge is to find an invariant. Unno et al. [2017] presented an inductive proof system tailored to $\mathrm{CHC}$ solving and applied it to relational verification. The search algorithms presented in these studies correspond to bounded model-checking with covering, and the cut-rules (i.e., (CoNs) rule in [Tellez and Brotherston 2020] and (APPLY $\perp) /($ ApplyP) rules in [Unno et al. 2017]) are used only when one needs to check whether an open leaf node is covered. Researchers have extended SMT solvers to efficiently handle recursive functions and applied them to verification of programs that manipulate structured data [Qiu et al. 2013; Reynolds and Kuncak 2015; Suter et al. 2010, 2011] These work however neither show a connection to modern software model checker nor discuss heuristics to find a good cut-formula using software model checking techniques.

Entailment checking. Many papers are devoted to give (efficient) automated theorem provers for entailment problems with inductive predicates [Brotherston 2005; Brotherston et al. 2011; Chin et al. 2012; Chu et al. 2015; Iosif et al. 2013; Ta et al. 2016, 2017] (in particular, of separation logic) and to find decidable fragments of the entailment problems [Berdine et al. 2004; Le et al 2017]. However, to our knowledge, there is no work that shows connection to modern software model checker. An interesting question is whether these developments and ideas are applicable to software model-checking. For example, global trace condition for cyclic proofs gives us a more flexible covering criterion than the naïve one used in most software model-checkers. Also, lemma discovery techniques developed for entailment checking [Enea et al. 2015; Ta et al. 2016, 2017] or general purpose inductive theorem proving [Bundy 2001] can be useful in software model checking. Conversely, it is interesting to investigate if Craig interpolation and other heuristics for finding a good cut-formula that have been studied in the software model checking community can be effectively applied as lemma discovery heuristics to inductive theorem proving.

\subsection{Software Model Checking}

Unified framework. Beyer et al. have been developing a configurable software model checker CPACHecker [Beyer et al. 2007, 2008; Beyer and Keremoglu 2011], which implements various software model checking algorithms [Beyer and Dangl 2020; Beyer et al. 2018; Beyer and Wendler 2012] in a unified framework in a configurable manner. Though our logical foundation also provides a unified framework, the level of abstraction is significantly different: we reconstruct well-known algorithms from a few simple and declarative principles, whereas CPACHECKER achieves the reconstruction via modular design and implementation of software model checking components.

Constraint logic programming. Constraint logic programming (CLP) has been used as a logical foundation of software model checking [Bjørner et al. 2015; Flanagan 2004; Podelski and Rybalchenko 2007]. They use CLP to model the target specification as well as the concrete or abstract execution semantics of the target program and then invoke a CLP solver to perform an actual proof-search such as symbolic execution, predicate abstraction, and abstraction refinement. By contrast, the logical foundation proposed in this paper aims at analyzing and comparing existing and unseen software model checking algorithms as different proof-search strategies in the same cyclic proof system. In other words, we provide a logical foundation for not only modeling but also solving safety verification problems.

Interpolating theorem provers. We presented declarative characterizations of interpolation-based frame refinement, where we regarded interpolating theorem provers as a black box. Their internal 
states and syntactic features (e.g., size and shape) of their outputs cannot be captured by the current cyclic-proof framework. We plan to investigate the following research questions: Is it possible to see the internal states as partial proofs? Is it possible to give a declarative characterization for practically "good" interpolants: those returned by PDR and beautiful interpolants [Albarghouthi and McMillan 2013].

Other approaches to invariant synthesis. Other approaches include constraint-based one [Colón et al. 2003; Sankaranarayanan et al. 2004] that uses $\exists \forall$-formula solvers and CEGIS [Solar-Lezama et al. 2006] that uses inductive synthesizers [Garg et al. 2016; Sharma et al. 2013]. The cyclic-proof framework needs further extensions to capture their internal states: for instance, for the latter we need to annotate each judgement in a partial proof with examples that are iteratively collected by the CEGIS procedure.

\section{CONCLUSION AND FUTURE WORK}

This paper establishes a tight connection between software model-checkers and cyclic-proof search strategies. It is worth emphasizing that our connection relates internal states of modern, sophisticated software model-checkers and partial proofs in the cyclic proof system. The logical viewpoint is useful for understanding model-checking procedures. A rich structure of (or strong constraints on) partial proofs allows us to reconstruct important model-checking procedures from a few simple principles: for example, PDR is a proof seach strategy following Principles 1, 3, 4 and 5. Furthermore the logical characterization of PDR significantly extends the scope of application, as discussed in section 7 .

The most important future work is an empirical study. An interesting question is about the efficiency of an alternative implementation of PDR, in which maximally conservative interpolants are computed in a different way. If it is comparable to the original PDR, the development of an efficient maximally-conservatively interpolating theorem prover would be of practical interest.

Future work in another direction is a study of model-checking procedures that are not covered by this paper, such as GPDR [Hoder and Bjørner 2012] and SpACER [Komuravelli et al. 2014] for safety verification of programs with first-order functions. This class of the verification problem is out of the scope of our framework: although it has an inductive characterization, it does not have the dual, coinductive characterization.

We are also interested in the development of new model-checkers using logical ideas. For example, the safety verification of programs with functions has an inductive characterization $(\mu R)(x) \vdash \alpha(x)$, whereas it has no coinductive characterisation. The logical analysis of the paper suggests that backward execution looks more natural (cf. Section 5), but empirical evaluation of this suggestion is left for future work.

\section{ACKNOWLEDGMENTS}

This work was supported by JST ERATO HASUO Metamathematics for Systems Design Project (No. JPMJER1603) and JSPS KAKENHI Grant Numbers JP20H05703, JP19K22842, JP20H04162, JP17H01720, and JP19H04084.

\section{REFERENCES}

Aws Albarghouthi and Kenneth L. McMillan. 2013. Beautiful Interpolants. In CAV'13 (LNCS), Vol. 8044. Springer, 313-329. https://doi.org/10.1007/978-3-642-39799-8_22

Thomas Ball, Byron Cook, Vladimir Levin, and Sriram K. Rajamani. 2004. SLAM and Static Driver Verifier: Technology Transfer of Formal Methods inside Microsoft. In Integrated Formal Methods. Springer, 1-20. https://doi.org/10.1007/9783-540-24756-2_1 
Thomas Ball, Rupak Majumdar, Todd Millstein, and Sriram K. Rajamani. 2001. Automatic predicate abstraction of C programs. In PLDI '01 (Snowbird, Utah, United States). ACM, 203-213. https://doi.org/10.1145/378795.378846

Thomas Ball and Sriram K. Rajamani. 2002. The SLAM project: debugging system software via static analysis. In POPL ' 02 (Portland, Oregon). ACM, 1-3. https://doi.org/10.1145/503272.503274

Josh Berdine, Cristiano Calcagno, and Peter W. O’Hearn. 2004. A Decidable Fragment of Separation Logic. In FSTTCS '04 (Chennai, India, 16-18) (LNCS), Vol. 3328. Springer, 97-109. https://doi.org/10.1007/b104325

Dirk Beyer and Matthias Dangl. 2020. Software Verification with PDR: An Implementation of the State of the Art. In TACAS '20. Springer, 3-21. https://doi.org/10.1007/978-3-030-45190-5_1

Dirk Beyer, Matthias Dangl, and Philipp Wendler. 2018. A Unifying View on SMT-Based Software Verification. fournal of Automated Reasoning 60, 3 (2018), 299--335. https://doi.org/10.1007/s10817-017-9432-6

Dirk Beyer, Thomas A. Henzinger, and Grégory Théoduloz. 2007. Configurable Software Verification: Concretizing the Convergence of Model Checking and Program Analysis. In CAV '07. Springer, 504-518. https://doi.org/10.1007/978-3540-73368-3_51

D. Beyer, T. A. Henzinger, and G. Theoduloz. 2008. Program Analysis with Dynamic Precision Adjustment. In ASE '08. IEEE, 29-38. https://doi.org/10.1109/ASE.2008.13

Dirk Beyer and M. Erkan Keremoglu. 2011. CPAchecker: A Tool for Configurable Software Verification. In CAV '11. Springer, 184-190. https://doi.org/10.1007/978-3-642-22110-1_16

D. Beyer and P. Wendler. 2012. Algorithms for software model checking: Predicate abstraction vs. Impact. In FMCAD '12. IEEE, 106-113.

Armin Biere, Alessandro Cimatti, Edmund Clarke, and Yunshan Zhu. 1999. Symbolic Model Checking without BDDs. In TACAS '99. Springer, 193-207. https://doi.org/10.1007/3-540-49059-0_14

Johannes Birgmeier, Aaron R. Bradley, and Georg Weissenbacher. 2014. Counterexample to Induction-Guided AbstractionRefinement (CTIGAR). In CAV'14. Springer, 831-848. https://doi.org/10.1007/978-3-319-08867-9_55

Nikolaj Bjørner, Arie Gurfinkel, Kenneth L. McMillan, and Andrey Rybalchenko. 2015. Horn Clause Solvers for Program Verification. In Fields of Logic and Computation II: Essays Dedicated to Yuri Gurevich on the Occasion of His 75th Birthday (LNCS), Vol. 9300. Springer, 24-51. https://doi.org/10.1007/978-3-319-23534-9_2

Aaron R. Bradley. 2011. SAT-based Model Checking Without Unrolling. In VMCAI '11 (Austin, TX, USA) (LNCS), Vol. 6538. Springer, 70-87. https://doi.org/10.1007/978-3-642-18275-4_7

James Brotherston. 2005. Cyclic Proofs for First-Order Logic with Inductive Definitions. In TABLEAUX '05 (LNCS), Vol. 3702. Springer, 78-92. https://doi.org/10.1007/11554554_8

James Brotherston, Richard Bornat, and Cristiano Calcagno. 2008. Cyclic Proofs of Program Termination in Separation Logic. In POPL '08. ACM, 101-112. https://doi.org/10.1145/1328438.1328453

James Brotherston, Dino Distefano, and Rasmus Lerchedahl Petersen. 2011. Automated Cyclic Entailment Proofs in Separation Logic. In CADE-23 (Wrocław, Poland). Springer, 131-146. https://doi.org/10.1007/978-3-642-22438-6_12

James Brotherston, Nikos Gorogiannis, and Rasmus L. Petersen. 2012. A Generic Cyclic Theorem Prover. In APLAS '12. Springer, 350-367. https://doi.org/10.1007/978-3-642-35182-2_25

J. Brotherston and A. Simpson. 2011. Sequent calculi for induction and infinite descent. fournal of Logic and Computation 21, 6 (2011), 1177-1216. https://doi.org/10.1093/logcom/exq052

Alan Bundy. 2001. The Automation of Proof by Mathematical Induction. In Handbook of Automated Reasoning, Vol. I. Elsevier, 845-911. https://doi.org/10.1016/b978-044450813-3/50015-1

Wei-Ngan Chin, Cristina David, Huu Hai Nguyen, and Shengchao Qin. 2012. Automated Verification of Shape, Size and Bag Properties via User-Defined Predicates in Separation Logic. Sci. Comput. Program. 77, 9 (2012), 1006-1036. https://doi.org/10.1016/j.scico.2010.07.004

Duc-Hiep Chu, Joxan Jaffar, and Minh-Thai Trinh. 2015. Automatic Induction Proofs of Data-structures in Imperative Programs. In PLDI '15 (Portland, OR, USA). ACM, 457-466. https://doi.org/10.1145/2737924.2737984

Alessandro Cimatti and Alberto Griggio. 2012. Software Model Checking via IC3. In CAV'12. Springer, 277-293. https: //doi.org/10.1007/978-3-642-31424-7_23

Alessandro Cimatti, Alberto Griggio, Sergio Mover, and Stefano Tonetta. 2014. IC3 Modulo Theories via Implicit Predicate Abstraction. In TACAS '14. Springer, 46-61. https://doi.org/10.1007/978-3-642-54862-8_4

Edmund M. Clarke, Orna Grumberg, Somesh Jha, Yuan Lu, and Helmut Veith. 2003. Counterexample-guided abstraction refinement for symbolic model checking. F. ACM 50, 5 (2003), 752-794. https://doi.org/10.1145/876638.876643

Michael A. Colón, Sriram Sankaranarayanan, and Henny B. Sipma. 2003. Linear Invariant Generation Using Non-linear Constraint Solving. In $C A V$ '03 (Chennai, India) (LNCS), Vol. 2725. Springer, 420-432. https://doi.org/10.1007/978-3-54045069-6_39

William Craig. 1957. Three Uses of the Herbrand-Gentzen Theorem in Relating Model Theory and Proof Theory. fournal of Symbolic Logic 22 (1957), 269-285. https://doi.org/10.2307/2963594 
Niklas Een, Alan Mishchenko, and Robert Brayton. 2011. Efficient Implementation of Property Directed Reachability. In FMCAD '11 (Austin, Texas). IEEE, 125-134.

Constantin Enea, Mihaela Sighireanu, and Zhilin Wu. 2015. On Automated Lemma Generation for Separation Logic with Inductive Definitions. In ATVA '15. Springer, 80-96. https://doi.org/10.1007/978-3-319-24953-7_7

Azadeh Farzan and Zachary Kincaid. 2016. Linear Arithmetic Satisfiability via Strategy Improvement. In IfCAI '16. AAAI Press, 735-743.

Azadeh Farzan and Zachary Kincaid. 2017. Strategy Synthesis for Linear Arithmetic Games. Proceedings of the ACM on Programming Languages 2, POPL, Article 61 (Dec. 2017), 30 pages. https://doi.org/10.1145/3158149

Cormac Flanagan. 2004. Automatic software model checking via constraint logic. Science of Computer Programming 50, 1 (2004), 253-270. https://doi.org/10.1016/j.scico.2004.01.006

Pranav Garg, Daniel Neider, P. Madhusudan, and Dan Roth. 2016. Learning Invariants Using Decision Trees and Implication Counterexamples. In POPL '16 (St. Petersburg, FL, USA). ACM, 499-512. https://doi.org/10.1145/2837614.2837664

Susanne Graf and Hassen Saïdi. 1997. Construction of Abstract State Graphs with PVS. In CAV '97 (Haifa, Israel, 22-25) (LNCS), Vol. 1254. Springer, 72-83. https://doi.org/10.1007/3-540-63166-6_10

Thomas A. Henzinger, Ranjit Jhala, Rupak Majumdar, and Kenneth L. McMillan. 2004. Abstractions from proofs. In POPL '04 (Venice, Italy). ACM, 232-244. https://doi.org/10.1145/964001.964021

Thomas A. Henzinger, Ranjit Jhala, Rupak Majumdar, and Grégoire Sutre. 2002. Lazy abstraction. In POPL '02 (Portland, Oregon). ACM, 58-70. https://doi.org/10.1145/503272.503279

Kryštof Hoder and Nikolaj Bjørner. 2012. Generalized Property Directed Reachability. In SAT '12 (Trento, Italy). Springer, 157-171. https://doi.org/10.1007/978-3-642-31612-8_13

Radu Iosif, Adam Rogalewicz, and Jiri Simacek. 2013. The Tree Width of Separation Logic with Recursive Definitions. In CADE-24. Springer, 21-38. https://doi.org/10.1007/978-3-642-38574-2_2

Ranjit Jhala and Rupak Majumdar. 2009. Software Model Checking. ACM Comput. Surv. 41, 4, Article 21 (Oct. 2009 ), 54 pages. https://doi.org/10.1145/1592434.1592438

Alexey Khoroshilov, Vadim Mutilin, Alexander Petrenko, and Vladimir Zakharov. 2010. Establishing Linux Driver Verification Process. In Perspectives of Systems Informatics. Springer, 165-176. https://doi.org/10.1007/978-3-642-11486-1_14

Daisuke Kimura, Koji Nakazawa, Tachio Terauchi, and Hiroshi Unno. 2020. Failure of Cut-Elimination in Cyclic Proofs of Separation Logic. Computer Software 37, 1 (2020), 1_39-1_52. https://doi.org/10.11309/jssst.37.1_39

Anvesh Komuravelli, Nikolaj Bjørner, Arie Gurfinkel, and Kenneth L. McMillan. 2015. Compositional Verification of Procedural Programs Using Horn Clauses over Integers and Arrays. In FMCAD ’15 (Austin, Texas). FMCAD Inc, 89-96. https://doi.org/10.1109/FMCAD.2015.7542257

Anvesh Komuravelli, Arie Gurfinkel, and Sagar Chaki. 2014. SMT-Based Model Checking for Recursive Programs. In $C A V$ '14 (LNCS), Vol. 8559. Springer, 17-34. https://doi.org/10.1007/978-3-319-08867-9_2

Anvesh Komuravelli, Arie Gurfinkel, and Sagar Chaki. 2016. SMT-Based Model Checking for Recursive Programs. Formal Methods in System Design 48, 3 (June 2016), 175-205. https://doi.org/10.1007/s10703-016-0249-4

Anvesh Komuravelli, Arie Gurfinkel, Sagar Chaki, and Edmund M. Clarke. 2013. Automatic Abstraction in SMT-Based Unbounded Software Model Checking. In CAV'13. Springer, 846-862. https://doi.org/10.1007/978-3-642-39799-8_59

Quang Loc Le, Makoto Tatsuta, Jun Sun, and Wei-Ngan Chin. 2017. A Decidable Fragment in Separation Logic with Inductive Predicates and Arithmetic. In CAV'17. Springer, 495-517. https://doi.org/10.1007/978-3-319-63390-9_26

Per Martin-Löf. 1971. Hauptsatz for the Intuitionistic Theory of Iterated Inductive Definitions. In Proceedings of the Second Scandinavian Logic Symposium. Studies in Logic and the Foundations of Mathematics, Vol. 63. Elsevier, 179 - 216. https://doi.org/10.1016/S0049-237X(08)70847-4

Yukihiro Masuoka and Makoto Tatsuta. 2021. Counterexample to cut-elimination in cyclic proof system for first-order logic with inductive definitions. CoRR abs/2106.11798 (2021). arXiv:2106.11798

Kenneth L. McMillan. 2006. Lazy Abstraction with Interpolants. In CAV'06 (Seattle, WA, USA, 17-20) (LNCS), Vol. 4144. Springer, 123-136. https://doi.org/10.1007/11817963

Andreas Podelski and Andrey Rybalchenko. 2007. ARMC: The Logical Choice for Software Model Checking with Abstraction Refinement. In Practical Aspects of Declarative Languages. Springer, 245-259. https://doi.org/10.1007/978-3-540-69611$7 \_16$

Xiaokang Qiu, Pranav Garg, Andrei Ştefănescu, and Parthasarathy Madhusudan. 2013. Natural Proofs for Structure, Data, and Separation. In PLDI '13. ACM, 231-242. https://doi.org/10.1145/2491956.2462169

Andrew Reynolds and Viktor Kuncak. 2015. Induction for SMT Solvers. In VMCAI '15 (LNCS), Vol. 8931. Springer, 80-98. https://doi.org/10.1007/978-3-662-46081-8_5

Sriram Sankaranarayanan, Henny B. Sipma, and Zohar Manna. 2004. Non-linear loop invariant generation using Gröbner bases. In POPL '04 (Venice, Italy). ACM, 318-329. https://doi.org/10.1145/964001.964028

Rahul Sharma, Saurabh Gupta, Bharath Hariharan, Alex Aiken, and Aditya V. Nori. 2013. Verification as Learning Geometric Concepts. In SAS '13. Springer, 388-411. https://doi.org/10.1007/978-3-642-38856-9_21 
Mary Sheeran, Satnam Singh, and Gunnar Stålmarck. 2000. Checking Safety Properties Using Induction and a SAT-Solver. In FMCAD '00. Springer, 127-144. https://doi.org/10.1007/3-540-40922-X_8

Armando Solar-Lezama, Liviu Tancau, Rastislav Bodik, Sanjit Seshia, and Vijay Saraswat. 2006. Combinatorial Sketching for Finite Programs. In ASPLOS XII (San Jose, California, USA). ACM, 404-415. https://doi.org/10.1145/1168857.1168907

Christoph Sprenger and Mads Dam. 2003. On the Structure of Inductive Reasoning: Circular and Tree-Shaped Proofs in the $\mu$ Calculus. In FoSSaCS '03. Springer, 425-440. https://doi.org/10.1007/3-540-36576-1_27

Philippe Suter, Mirco Dotta, and Viktor Kuncak. 2010. Decision procedures for algebraic data types with abstractions. In POPL '10 (Madrid, Spain). ACM, 199-210. https://doi.org/10.1145/1706299.1706325

Philippe Suter, Ali Sinan Köksal, and Viktor Kuncak. 2011. Satisfiability modulo recursive programs. In SAS '11 (Venice, Italy) (LNCS), Vol. 6887. Springer, 298-315. https://doi.org/10.1007/978-3-642-23702-7_23

Quang-Trung Ta, Ton Chanh Le, Siau-Cheng Khoo, and Wei-Ngan Chin. 2016. Automated Mutual Explicit Induction Proof in Separation Logic. In FM '16. Springer, 659-676. https://doi.org/10.1007/978-3-319-48989-6_40

Quang-Trung Ta, Ton Chanh Le, Siau-Cheng Khoo, and Wei-Ngan Chin. 2017. Automated Lemma Synthesis in SymbolicHeap Separation Logic. Proceedings of the ACM on Programming Languages 2, POPL, Article 9 (Dec. 2017), 29 pages. https://doi.org/10.1145/3158097

Gadi Tellez and James Brotherston. 2020. Automatically Verifying Temporal Properties of Pointer Programs with Cyclic Proof. Journal of Automated Reasoning 64, 3 (2020), 555-578. https://doi.org/10.1007/s10817-019-09532-0

Takeshi Tsukada and Hiroshi Unno. 2021. Software Model-Checking as Cyclic-Proof Search. arXiv:2111.05617

Hiroshi Unno, Sho Torii, and Hiroki Sakamoto. 2017. Automating Induction for Solving Horn Clauses. In $C A V$ '17. Springer, 571-591. https://doi.org/10.1007/978-3-319-63390-9_30

Yakir Vizel and Arie Gurfinkel. 2014. Interpolating Property Directed Reachability. In CAV '14. Springer, 260-276. https: //doi.org/10.1007/978-3-319-08867-9_17 